\title{
Das Versprechen der Architektur: Schaffen Multireligiöse Räume Toleranz?
}

\author{
Marian Burchardt (iD · Johanna Häring
}

Eingegangen: 27. Januar 2021 / Überarbeitet: 28. Mai 2021 / Angenommen: 23. Juni 2021 / Online publiziert: 23. Juli 2021

(C) Der/die Autor(en) 2021

Zusammenfassung Während interreligiöse Dialoge durch ikonische architektonische Projekte eine größere öffentliche Sichtbarkeit und mediale Aufmerksamkeit erlangt haben, ist deren breitere gesellschaftliche Wahrnehmung und Wirkung weitestgehend unerforscht. Der Beitrag untersucht gruppenspezifische Wahrnehmungen interreligiöser Beziehungen und Positionierungen zu interreligiösen Dialogen. Im Zentrum der Analyse stehen dabei die Debatten zum Berliner House of One, einem derzeit im Bau befindlichen multireligiösen Gebäude. Unter Rückgriff auf soziologische Konzeptualisierungen zum Verhältnis von Religion, Macht und Stadtraum untersucht der Beitrag, welche unterschiedlichen sozialen Positionen religiöser Akteur*innen (Protestant*innen, Alevit*innen und Muslim*innen) in den kulturellen Hierarchien urbaner Räume sich in solchen Wahrnehmungen dokumentieren. Wir argumentieren, dass die in diskursiven Aushandlungsprozessen zu interreligiösen Dialogen vorgenommenen Positionierungen solche kulturellen Hierarchien auf besondere Weise hervortreten lassen und damit auch auf blinde Flecken des DialogParadigmas aufmerksam machen. Der Beitrag basiert auf Gruppendiskussionen und Einzelinterviews.

Schlüsselwörter Religiöse Vielfalt · Interreligiöser Dialog · Stadtraum · Stadtsoziologie $\cdot$ Architektur $\cdot$ Berlin $\cdot$ House of One $\cdot$ Stadtplanung

Marian Burchardt $(\varangle) \cdot$ Johanna Häring Universität Leipzig, Beethovenstr. 15, 04107 Leipzig, Deutschland

E-Mail: marian.burchardt@uni-leipzig.de

Johanna Häring

E-Mail: johanna-haering@gmx.de 


\title{
The promise of architecture: Do multi-religious spaces promote tolerance?
}

\begin{abstract}
While interreligious dialogues have gained greater public visibility and media attention through iconic architectural projects, their broader social perception and impact remain mostly unexplored. This article examines group-specific perceptions of interreligious relations and positioning towards interreligious dialogues. At the center of the analysis are the debates on House of One, a multi-religious building currently under construction in Berlin. Based on sociological conceptualizations of the relationship between religion, power and urban space, the article examines how such perceptions document the divergent social positions of religious actors (Protestants, Alevis and Sunni Muslims) within the cultural hierarchies of urban space. We argue that the positionings made in discursive negotiations of interreligious dialogues articulate such cultural hierarchies and that they also draw attention to blind spots of the dialogue paradigm. The article is based on group discussions and individual interviews.
\end{abstract}

Keywords Religious diversity · Interreligious dialogue · Urban space · Urban sociology $\cdot$ Architecture $\cdot$ Berlin $\cdot$ House of One $\cdot$ Urban planning

\section{Einleitung}

Im Jahre 2011 beschlossen Vertreter einer protestantischen, einer jüdischen und einer muslimischen Gemeinde, an prominenter Stelle im Berliner Stadtbezirk Mitte ein Gebäude zu errichten, das unter einem Dach eine Kirche, Synagoge und Moschee vereinen soll. Seither zirkulierten Bilder und Visualisierungen des architektonischen Entwurfs in transnationalen Öffentlichkeiten. Dabei wurde der Entwurf bewertet und kommentiert und häufig ob seiner affektiven Strahlkraft gepriesen. Er wurde 2015 auf der Architektur-Biennale in Chicago und 2017 im Pariser Kulturzentrum Centquartre (2017) ${ }^{1}$ ausgestellt, und 2020 erwarb das prestigereiche Londoner Victoria and Albert Museum für Design ein Modell für seine Sammlung. Die Organisatoren zeigten den Entwurf zudem in einem temporären Pavillon am Bauort, im Rahmen verschiedener an Kinder gerichteten Aktionen unter dem Titel ,Young House of One“ (2017), in einer Vernissage, die eigens der Präsentation des Baumaterials (gebrannte Ziegel) gewidmet war (2020), und bei zahlreichen weiteren Gelegenheiten.

In öffentlichen Debatten wird dabei architektonischen Projekten wie dem House of One häufig die Fähigkeit zugeschrieben, durch Begegnung und den Abbau von gruppenbezogenen Vorurteilen interreligiöse Beziehungen positiv zu beeinflussen und damit zu friedlicher Koexistenz in einer von Segregation, Rassismen und Gewalt geprägten Gesellschaft beizutragen. Insofern trägt Architektur schon im Modellstadium dazu bei, Idee und Praxis des interreligiösen Dialogs, als deren materialer Ausdruck das House of One verstanden wird, zu popularisieren. Sie birgt damit in

\footnotetext{
1 https://house-of-one.org/de/news/house-one-paris.
} 
sich das Versprechen, dem Bemühen um friedliche Koexistenz materiale Form und ästhetischen Ausdruck zu geben.

Unser Beitrag hat das Ziel, eine Reihe von unausgesprochenen Annahmen, auf denen solche Zuschreibungen basieren, zu hinterfragen, insbesondere die Vorstellungen, dass interreligiöser Dialog per se gut sei, dass religiöse Gemeinschaften unter ähnlichen oder vergleichbaren Voraussetzungen in ihn eintreten und vor allem dass er tatsächlich in der Lage ist, wechselseitige Wahrnehmungen und kulturelle Grenzziehungen (Lamont und Molnár 2002) innerhalb von Stadtbevölkerungen positiv zu beeinflussen. Inwieweit dies der Fall ist, ist jedoch soziologisch bisher kaum erforscht worden. Unter Rückgriff auf soziologische Konzeptualisierungen zum Verhältnis von interreligiösen Dialogen, Macht und Stadtraum untersucht unser Beitrag daher am Beispiel des House of One, wie interreligiöse Dialoge gesellschaftlich wahrgenommen werden und welche unterschiedlichen sozialen Positionen religiöser Akteur*innen in den kulturellen Hierarchien urbaner Räume sich in deren Wahrnehmungen dokumentieren. Wir argumentieren, dass die in diskursiven Aushandlungsprozessen zu interreligiösen Dialogen vorgenommenen Positionierungen solche kulturellen Hierarchien auf besondere Weise hervortreten lassen und damit auch auf blinde Flecken des Dialog-Paradigmas aufmerksam machen. Wie im Abschn. 5 im Detail erläutern, liegen diese blinden Flecken in erster Linie in den multiplen, nicht antizipierten Gegenreaktionen, mit denen interreligiöse Dialoge - als notwendigerweise reglementierte Verfahren - konfrontiert sind. Wie der Lichtstrahl in einem Prisma bricht sich das Versprechen der Architektur damit an den vielfältigen Fragmentierungen der Stadtgesellschaft.

\section{Das House of One}

Die Idee zur Errichtung des House of One entstand im Kontext der Rückübertragung des Baugrundstückes auf dem Berliner Petriplatz an die lokale Kirchgemeinde in den 1990er-Jahren. Auf dem Platz, der als einer der Gründungsorte Berlins gilt, standen seit 1230 fünf verschiedene Kirchgebäude. Das letzte dieser Gebäude wurde während des zweiten Weltkrieges stark beschädigt, woraufhin es im Jahre 1960 zum Abriss freigegeben wurde. Der Platz wurde anschließend gemäß den Vorgaben der modernistischen Stadtplanung in vielspurige Straßenensembles eingefügt und ist derzeit von multiplen Stadterneuerungsvorhaben umgeben.

Träger des Projekts war bis zur Gründung der Stiftung House of One - Bet- und Lehrhaus Berlin im Jahre 2016 der gleichnamige Verein, in dem das Land Berlin, von protestantischer Seite die Gemeinde St. Petri, von jüdischer Seite das AbrahamGeiger Kolleg Potsdam sowie von muslimischer Seite das Forum für interkulturellen Dialog vertreten sind. Die Beteiligung dieser, der Bewegung des umstrittenen türkisch-amerikanischen Laienpredigers Fetullah Gülen nahestehenden, Organisation hat von Beginn an immer wieder öffentliche Kritik - vor allem, aber nicht ausschließlich, aufseiten der muslimischen Dachverbände - hervorgerufen und 2019 zum öffentlich inszenierten Austritt eines der Hauptsponsoren, des Unternehmens Dussmann, aus dem Stiftungsrat geführt. Im Jahre 2012 lobte der Verein einen Ar- 
Abb. 1 Entwurf von Kuehn und Malvezzi, Außenansicht

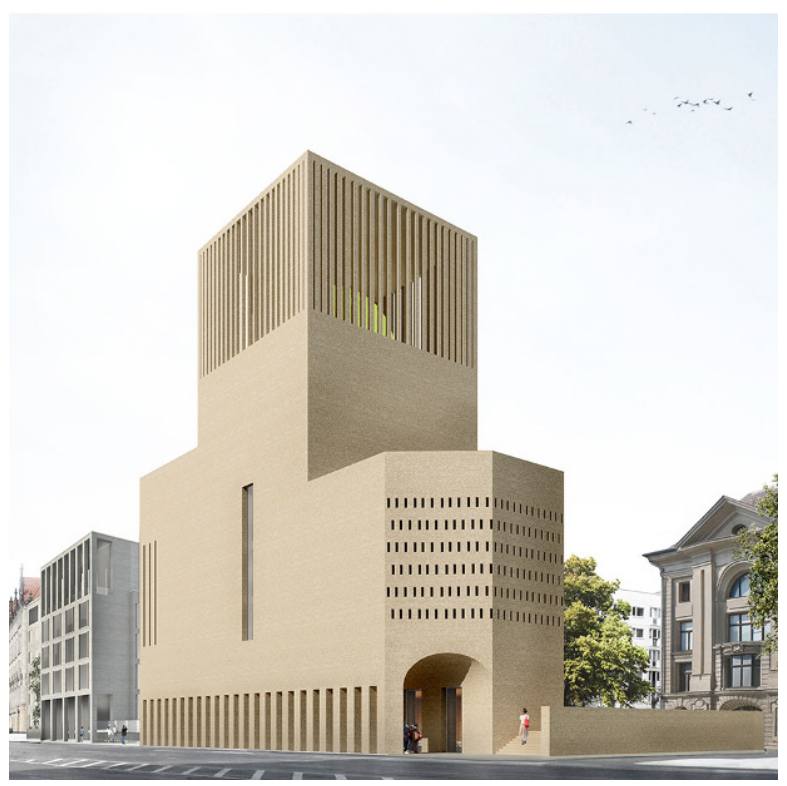

chitekturwettbewerb aus, aus dem der Entwurf des Architekturbüros Kuehn und Malvezzi als Sieger hervorging. (Abb. 1 und 2).

Seither engagieren sich die beteiligten religiösen Akteur*innen in einer Vielzahl von Aktivitäten wie interreligiösen Meditationen, Informationsveranstaltungen, interreligiösen Stadtrundgängen, gemeinsamen Friedensgebeten und Trauerandachten, die häufig auf jüngere Ereignisse wie etwa terroristische Attentate Bezug nehmen. Gleichzeitig popularisieren sie das Projekt über öffentliche Diskussionsveranstaltungen an Orten wie dem Stadion der Bundesliga-Fußballmannschaft Union Berlin, dem Polizeipräsidium und dem Deutschen Theater, wo jeweils zielgruppenspezifische Aspekte (Zusammenhalt, Gewalt usw.) thematisiert werden. Während damit das House of One bereits vor seinem Bau in den Stadtraum hineinwirkt, werden diese Aktivitäten durchweg als Events gerahmt und visuell über Fotos und Videomitschnitte dokumentiert und ,bebildert“, die über die Social-Media-Kanäle des House of One geteilt werden und die mediale Aufmerksamkeit in transnationalen Öffentlichkeiten verstärken. Die durch die COVID19-Pandemie erzwungene Verlagerung von Events in virtuelle Räume hat die digitale Eventisierung des House of One dabei noch verstärkt.

Diese Eventisierung und Mediatisierung des House of One sind zentrale Pfeiler der ursprünglich anvisierten Finanzierung des mit $€ 47$ Mio. veranschlagten Baus über eine als Crowd-Funding organisierte Spendenkampagne. Im Rahmen der Kampagne können Spender*innen symbolisch Backsteine für jeweils $€ 10$ erwerben und somit am House of One ,mitbauen“. Allerdings reichte die Spendenbereitschaft 
Abb. 2 Entwurf von Kuehn und Malvezzi, Innenansicht

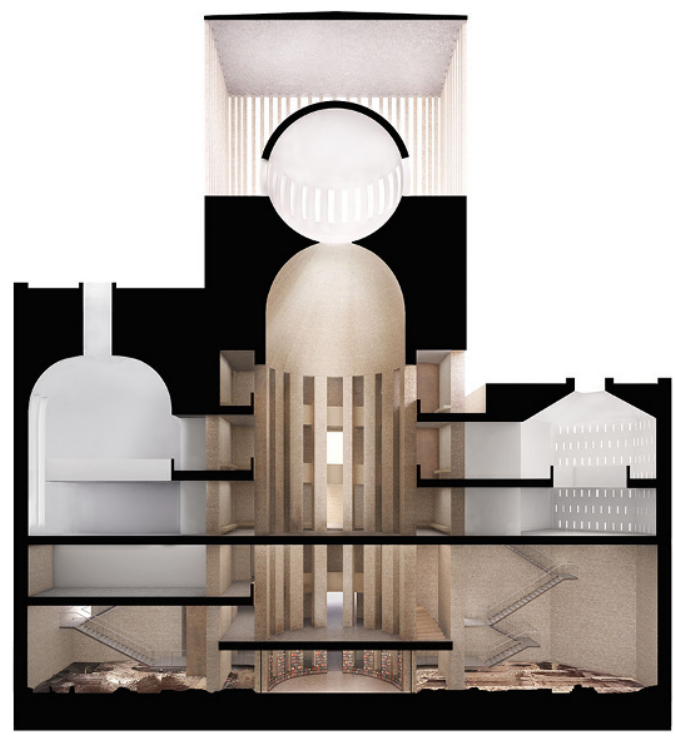

nicht aus, um das Projekt zu realisieren, so dass das Gebäude nun mehrheitlich durch staatliche Gelder finanziert wird. ${ }^{2}$

Seit der Gründung der Initiative hat das House of One weltweit mediale Aufmerksamkeit erregt und steht in regelmäßigen Abständen im Fokus globaler Leitmedien wie des Guardian ${ }^{3}$, der BBC ${ }^{4}$ und Al Jazeera. ${ }^{5}$ Dabei spielen nicht zuletzt historische Verweise eine zentrale Rolle, in denen auf die Geschichte Berlins, des Zweiten Weltkrieges und des Holocausts, die Geschichte des Kalten Krieges und des ostdeutschen Kommunismus wie auch auf die Geschichte des Mauerfalls und Berlins Status als gegenwärtigem Leuchtturm von Multikulturalismus und Kosmopolitismus Bezug genommen wird. In den entsprechenden Diskursen werden Berlin und das House of One als Kristallisationspunkte interkultureller Begegnung und Verständigung adressiert, die beispielhaft für die Möglichkeit des Überwindens politischer, kultureller und religiöser Grenzen stehen. Aus dem Hintergrund einer wechselvollen Geschichte von Gewalt und Menschenrechtsverletzungen wird in den Mediendiskursen das Versprechen einer friedvollen Zukunft abgeleitet, für das die Architektur des House of One in spezifischer Weise einsteht.

\footnotetext{
2 Die Förderung erfolgt unter anderen aus den Mitteln des Programms „Nationaler Städtebau“. Im Rahmen eines diesbezüglichen Wettbewerbs war das Land Berlin mit seinem Antrag auf Förderung des House of One im Jahre 2016 erfolgreich.

3 Siehe https://www.theguardian.com/world/shortcuts/2014/jun/25/berlin-house-of-one-unity-christianmuslim-jew.

4 Siehe https://www.bbc.com/news/magazine-27872551.

5 Siehe https://www.youtube.com/watch?v=6105cjQTfco\&feature=youtu.be.
} 


\section{Interreligiöse Beziehungen im Stadtraum: Theoretische Ansätze}

Die zentrale These unseres Beitrages ist, dass interreligiöse Dialoge nur dann die ihnen von den Befürworter*innen unterstellten Effekte der Förderung urbaner Koexistenz hervorbringen können, wenn sie tatsächlich in urbanen Räumen Resonanz erzeugen. Unter dem Begriff des „Dialog-Paradigma“, welchem interreligiöse Dialoge u.E. folgen, können dabei all jene Aktivitäten zusammengefasst werden, die sich bei bewusster Ausklammerung von Machtgefällen unter den beteiligten Akteur*innen um die Lösung von Konflikten durch deliberative Verfahren bemühen. So argumentiert etwa Amir-Moazimi (2011, S. 13): „Although clearly diverse in scope and plural in the approaches, one common feature in these initiatives is their rising inscription into a teleological rationality that aims at solving societal, political and partly even economic problems and conflicts."

Wir nähern uns den möglichen Wirkungen interreligiöser Architekturprojekten wie dem House of One und den durch sie gestifteten Diskursen auf die Wahrnehmung interreligiöser Beziehungen, indem wir zwei, sich bisher weitgehend isoliert entwickelnde, soziologische Ansätze miteinander in Beziehung setzen: Forschungen zu religiöser Vielfalt in Städten (1) und sozialwissenschaftliche Studien zu interreligiösen Dialogen (2). Beide Forschungsrichtungen tragen wesentliche Elemente zum theoretischen Verständnis interreligiöser Beziehungen und Kategorisierungen bei, die jedoch erst in ihrer Synthese die Wirkungsweise multireligiöser Räume erhellen. Forschungen zu religiöser Vielfalt und urbanen Räumen fokussieren auf Strukturen und Alltagswahrnehmungen religiöser Differenz, haben dabei aber bisher kaum die affektiven Effekte von interreligiösen Dialogen und deren architektonischen Projekten in den Blick genommen. Studien zu interreligiösen Dialogen wiederum haben deren Bedeutungsvielfalt, Handlungsrepertoires und politische Bedeutungszunahme aufzeigen können, beleuchten aber bisher kaum die Bedingungen, unter denen solche Dialogprozesse gesellschaftliche Resonanz in Stadtgesellschaften erzeugen. ${ }^{6}$

\subsection{Religiöse Vielfalt und interreligiöse Beziehungen im urbanen Raum}

An der Schnittstelle von Stadtsoziologie und Religionssoziologie hat sich innerhalb der letzten 20 Jahre ein Forschungsfeld etabliert, das die multiplen Beziehungen und wechselseitigen Prägungen von religiösen Zugehörigkeiten und Praktiken einerseits und urbanen Räumen andererseits ins Zentrum der Aufmerksamkeit gerückt hat. Die den meisten Studien zugrunde liegende Kernthese ist dabei, dass die Vorstellung einer durch Urbanisierungsprozesse vorangetriebenen Säkularisierung in den durch Migrationsprozesse und religiöse Innovationen sich pluralisierenden Großstädten westlicher Einwanderungsgesellschaften die soziale Wirklichkeit nicht mehr angemessen abbildet. Urbane Räume sind stattdessen durch eine fortwährende Diversifizierung gekennzeichnet, die unter Rückgriff auf einen populären Begriff von Steven Vertovec (2007) als religiöse „Super-Diversität“ gefasst werden kann (siehe Burchardt und Becci 2016). Diese Super-Diversität umfasst dabei sowohl die Vielfalt religiöser Gruppen wie auch Differenzen in Bezug auf die Relevanz von Religion

\footnotetext{
6 Ausnahmen sind Griera $(2019,2020)$.
} 
und Säkularität im Alltagsleben unterschiedlicher Bevölkerungsgruppen, die Peter L. Berger (2014) im Konzept einer zweifachen Pluralisierung zusammengefasst hat.

Vier konzeptuelle Dimensionen sind mit Blick auf das soziologische Verständnis der Manifestation und der sozialen Auswirkungen dieser religiösen Diversifizierung urbaner Räume von zentraler Bedeutung: Zugehörigkeit, Verräumlichung, Sichtbarkeit und Materialität. Dabei übergreifen diese Dimensionen die Gesamtheit der Formen und Erscheinungsweisen urbaner Regulierungen von Religion, wie die Planung religiöser Gebäude (etwa Design und Größe), die dazugehörigen infrastrukturellen Regelungen (bzgl. Parkraum, Lautstärke von Veranstaltungen, Verbot von MuezzinRufen usw.), die Genehmigung von Straßensperrungen für religiöse Feste und damit zusammenhängende Wahrnehmungen von „Belästigung“, die Hygienevorschriften bei rituellen Schlachtungen, Friedhöfe, öffentliche interreligiöse Zeremonien mit politischer Beteiligung wie auch die Auseinandersetzungen um religiöse Bekleidungen wie die Kippa und die Niqab und damit einhergehende Stigmatisierungen und Diskriminierungen.

Erstens sind über die Erforschung urbaner Religion neue Auseinandersetzungen um gesellschaftliche Zugehörigkeit und Teilhabe in den Blick geraten, innerhalb derer die kulturellen Hierarchien, die Städte prägen, sichtbar gemacht worden und zum Gegenstand von Konflikten geworden sind. Solche Hierarchien zeigen sich unter anderem an gestuften Formen des Zugangs zu öffentlichen Räumen, etwa im Kontext der Beantragung und Durchführung religiöser Feste und Zeremonien auf Plätzen und Straßen, oder der Selbstverständlichkeit, mit der sich Personengruppen im öffentlichen Raum über Kleidung und Symbole als einer spezifischen Gemeinschaft zugehörig markieren.

Zweitens geht religiöse Diversifizierung mit spezifischen Formen von Verräumlichung und Territorialität einher. Zentral ist dabei die raumsoziologische Prämisse, Räume nicht als gegeben, sondern als über Raumstrategien und raumbildende Praktiken im Kontext ungleicher Machtverhältnisse sozial erzeugt zu begreifen (Löw 2001). Im Ergebnis solcher Raumstrategien entstehen urbane Regime, die Zugang zu Räumen rechtlich kodifizieren und bürokratischen Normen unterwerfen, gewünschte Handlungs- und Selbstdarstellungsformen prämieren und urbane Interaktionsordnungen strukturieren (Griera und Burchardt 2020). Säkulare und religiöse Raumstrategien kristallisieren sich dann in Raumstrukturen, welche spezifische (religiöse) Praktiken sozialer Akteur*innen ermöglichen und sanktionieren, während sie andere ausschließen.

Als hierarchisch strukturierte Konfigurationen von menschlichen Akteur*innen, Architekturen und Infrastrukturen sind Städte sozio-materiale Gebilde, in denen religiöse Vielfalt also räumlich strukturiert ist und in denen religiöse und andere Kollektive um die Präsenz in und den Zugang zu Räumen - als vermeintlich knappen Gütern - konkurrieren bzw. sich wechselseitig als konkurrierend wahrnehmen und damit in Aushandlungen über kulturelle Definitionen und Bewertungen urbaner öffentlicher Räume (als „,bürgerlich“, ,,migrantisch“, ,,lebenswert“, ,,heruntergekommen“ usw.) eintreten (Knott et al. 2016, S. 127). Auch die Governance religiöser Vielfalt vollzieht sich deshalb in Städten vor allem über Raumzuweisungen und asymmetrisch strukturierte Raumnutzungen, etwa mit Blick auf den Bau religiöser Gebäude wie Moscheen, Synagogen oder Tempel, die Nutzung von Straßen, Plät- 
zen und Stadien für religiöse Prozessionen (etwa die schiitische Matam-Prozession, siehe Astor et al. 2018) oder Versammlungen (zur Papstmesse im Berliner Olympiastadion, siehe Knoblauch 2021).

Eng verbunden mit den Formen der Verräumlichung religiöser Vielfalt, die sich aus dem Zusammenspiel religiöser Raumstrategien - etwa derjenigen religiöser Minderheiten wie Muslime - und urbanen Regimen ergibt, stehen - drittens - Fragen der Sichtbarkeit von Religion (Garbin 2013; Kuppinger 2014). Dabei muss Sichtbarkeit als soziologische Kategorie, wie Brighenti (2007) deutlich herausstellt, immer mit Blick auf Anerkennung einerseits und soziale Kontrolle andererseits als zwei diametral entgegengesetzte Konsequenzen konzipiert werden.

So ist etwa die Ansiedlung von Moscheen migrantischer muslimischer Gemeinden in urbanen Peripherien als Form ihrer Unsichtbarmachung interpretiert worden und die Errichtung von Moscheen mit religiös konnotierter und entsprechend erkennbarer Architektur an zentralen Orten als Sichtbarmachung (Burchardt 2017; Cesari 2005; Göle 2011). Damit einher geht die Deutung architektonischer Visibilisierung und urbaner Prominenz als symbolische und politische Anerkennung von migrantischen religiösen Gruppen als gleichberechtigte und dauerhafte Bestandteile der Stadtgesellschaften (Knowles 2013). Das „Recht auf Stadt“ (Lefebvre 2016) dokumentiert sich hier als Recht auf angemessene architektonische und urbane Prominenz. Da Architektur, wie Fischer (2017) argumentiert, ein auf spezifische Weise „schweres“ und damit auch träges Medium des Sozialen ist, wird die Dauerhaftigkeit religiöser Pluralität und deren Anerkennung gerade an architektonischen Formen gesellschaftlich registriert. Umgekehrt zeigt die, in erster Linie über mediale Diskurse hergestellte, „Hypervisibilität“ von Niqab-Trägerinnen in westlichen urbanen Räumen auch, wie neue Formen von sozialer Kontrolle durch Sichtbarkeit vermittelt werden (Burchardt und Griera 2019). Die Akzentuierung von Sichtbarkeit kann also sowohl durch Abweichung von gesellschaftlich dominanten Normalitätsunterstellungen vermittelt und strategisch eingesetzt werden wie auch zum Ziel der Affirmation kultureller Normalitäten und Leitbilder. So zielt das House of One im Kern auf die Sichtbarmachung interreligiöser Begegnungen und Dialoge.

Aus solchen Auseinandersetzungen um öffentliche Sichtbarkeit ergeben sich - viertens - unmittelbar soziologische Fragen zur Rolle von Materialität in der Strukturierung urbaner Religion. Unter Bezugnahme auf Goffman (1959), Studien zu materialer Kultur, Latours objektzentrierter Soziologie (Latour 2001) und Konzepten aus dem Umfeld des neuen Materialismus (Coole und Frost 2010) haben architektursoziologische und stadtsoziologische Forschungen sich zunehmend auch mit der materialen Verfasstheit urbaner religiöser Formen beschäftigt und dabei auf die eigensinnigen und produktiven Verflechtungen von sozialem Sinn und Materialität verwiesen (Duttweiler 2017; Karstein und Schmidt-Lux 2017; Lanz 2016). Kultursoziolog*innen wie Geneviève Zubrzycki (2016) und Tia DeNora (2000) konzipieren dabei das Verhältnis von Objekten und deren physischen Eigenschaften über den Begriff der affordance, mit dem sie darauf verweisen, dass Objekte durch die „Bündelung“ physischer Eigenschaften jeweils nur spezifische religiöse Praktiken und Diskurse aus den umfassenderen kulturellen Repertoires religiöser Traditionen ermöglichen oder befördern (siehe auch Keane 2007). 


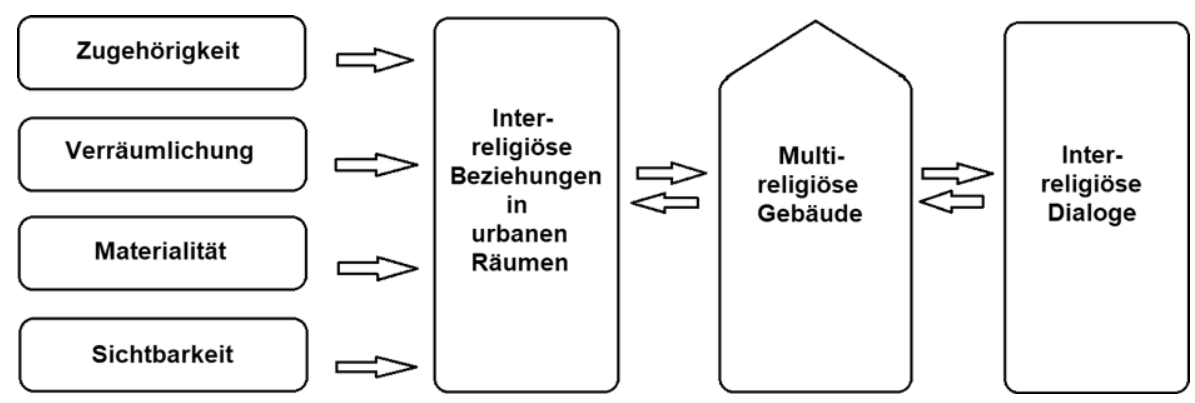

Abb. 3 Religiöse Vielfalt in urbanen Räumen: (Wechsel-)Beziehungen und Einflüsse (eigene Darstellung)

Innerhalb der Religionswissenschaft ist der material turn im Paradigma der material religion ausformuliert worden mit der zentralen Forderung, materiale Artefakte nicht lediglich als physische Ausdrucksformen und Trägerinnen fertiger und abgeschlossener Bedeutungsgehalte zu konzipieren, sondern als an der Produktion von kulturellen Bedeutungen beteiligt zu betrachten (Meyer 2011). Materiale religiöse Artefakte verkörpern die Beziehungen zwischen Gläubigen und dem Heiligen und markieren kulturelle Grenzen (Knott 2015). Sie wirken als Kristallisationspunkte für kollektive Identitäten und rufen spezifische historische Narrative und kollektive Erinnerungen auf. Vermittelt über ihre öffentliche Sichtbarkeit in urbanen Räumen können sie dabei auf unterschiedliche Art und Weise „gelesen“ (Knott et al. 2016, S. 126) werden. Je nach Perspektive kann eine katholische Kirche in Berlin von Anwohner*innen als kulturelles Erbe, als Ort ästhetischer Erfahrung oder spiritueller Erbauung, als touristische Sehenswürdigkeit, als bauliches Testament einer historischen Minderheit, als unauffällige Vergegenwärtigung einer säkularen kulturellen Normalität oder als auffälliges Symbol des eigenen Minderheitenstatus (etwa für Alevit*innen und Muslim*innen) wahrgenommen und interpretiert werden. Jede dieser Lesarten, so unser Argument, ist mit spezifischen Positionierungen in religiös-weltanschaulichen Feldern ${ }^{7}$ und dem Feld ethnischer Zugehörigkeit, das religiöse Kategorisierungen zutiefst prägt, verbunden (Brubaker 2017). Positionierungen im religiös-weltanschaulichen Feld und ethnische Zugehörigkeit unterliegen wiederum spezifisch stadträumlichen Prägungen. (Abb. 3).

Jede dieser vier Dimensionen ist offensichtlich für ein Verständnis der sozialen Konstruktion und der gesellschaftlichen Rolle von multi-religiösen architektonischen Projekten wie dem House of One von zentraler Bedeutung. ${ }^{8}$ Aufgrund seiner zentralen stadträumlichen Positionierung verleiht das House of One der Idee der interreligiösen Verständigung eine massive öffentliche Sichtbarkeit, die durch die Ikonizität des architektonischen Entwurfs weiter verstärkt wird (Sklair 2010). Als soziale und kommunikative Konstruktion (Steets 2015) laufen dabei im House of One verschiedene räumliche Arrangements und Skalen ineinander (innere baulich-

\footnotetext{
7 Zum Begriff des religiös-weltanschaulichen Feldes im Anschluss an Bourdieus Feldtheorie siehe Karstein (2013).

8 Mit dem Begriff der sozialen Konstruktion von Architektur folgen wir weitgehend dem wissenssoziologischen Ansatz von Silke Steets (2015), der uns maßgeblich inspiriert hat.
} 
materiale Gestaltung, stadträumliche Platzierung und die transnationale Zirkulation des Modells und dazugehörige Diskurse). Die durch die räumliche Platzierung vermittelte Sichtbarkeit unterstützt dabei die Popularisierung interreligiöser Dialoge und der ihnen zugrunde liegenden Vorstellung friedlicher interreligiöser Koexistenz.

Dabei verstärkt das spektakuläre skulpturale Design die öffentliche Aufmerksamkeit für das Gebäude, was wiederum an der Produktion von dessen Ikonizität mitwirkt. In ihrem bahnbrechenden Buch haben Bartmanski und Alexander (2012) die Wirkungsweise von Ikonizität folgendermaßen konzipiert: „Objects become icons when they have not only material force but also symbolic power. Actors have iconic consciousness when they experience material objects, not only understanding them cognitively or evaluating them morally, but feeling their sensual, aesthetic force" (2012, S. 1). Dass von dem Entwurf des House of One eine ikonische Kraft ausgeht, lässt sich an einer Vielzahl von Stellungnahmen ablesen, innerhalb derer sich affektive Bindungen von Individuen nicht nur zum Entwurf und seiner ästhetischen Sprache, sondern auch an die Idee einer durch ein materiales Objekt gestifteten friedlichen Welt artikulieren. ${ }^{9}$ Es ist die Möglichkeit dieser affektiven Bindung, die wir im Begriff des „,Versprechens der Architektur“ fassen.

Dass ikonische religiöse Bauprojekte häufig zutiefst umstritten sind, zeigt dabei, dass dieses Versprechen sehr unterschiedlich wahrgenommen wird. So belegen etwa die Debatten um die Wiedererrichtung der Leipziger Universitätskirche (SchmidtLux 2017), der Potsdamer Garnisonskirche (Hafner et al. 2018) und des Kreuzes auf dem Berliner Humboldtforum (Steets 2017), dass es hierbei nicht nur um Vielfalt, sondern im Gegenteil häufig auch um geschichtspolitisch kontroverse Inszenierungen religiösen und nationalen Kulturerbes geht. Was für die einen Versprechen, ist für andere ganz deutlich eine Bedrohung. Damit ist klar, dass Sichtbarkeit kein Garant für allgemeine Zustimmung ist, denn Sichtbarkeit verstärkt auch die Möglichkeit negativer Bewertung, wie wir in unserer empirischen Analyse zeigen werden.

\subsection{Interreligiöse Dialoge}

In der soziologischen Forschungsliteratur zum Verhältnis von Religion und Stadt rücken allmählich multireligiöse Räume ins Zentrum der Aufmerksamkeit. Multireligiöse Räume wie etwa das Haus der Religionen im Bern, der Campus der Religionen in Wien, das Haus der Religionen und Kulturen in München und das House of One sind eng verbunden mit dem Aufstieg des interreligiösen Dialogs als sozialem Modus der Binnenstrukturierung des religiösen Feldes und der Governance von Religion. Als feldspezifische Artikulation des Dialog-Paradigmas, das mittlerweile als „,politische Technik“ (Tezcan 2012, S. 35) weltweit in Prozessen der Friedenskonsolidierung (peace-building), der Bearbeitung ethnischer und zwischenstaatlicher Konflikte und der Bewältigung kollektiver Traumata zum Einsatz

\footnotetext{
9 In einer weiteren Komponente unseres Forschungsprojekts haben wir ca. 1000 Kommentare zum House of One in sozialen Medien (Facebook, Twitter und Instagram) gesammelt und hinsichtlich ihrer emotionalaffektiven Dimensionen und Gehalte codiert. Dieser Aspekt geht nicht in diesen Beitrag ein, belegt aber deutlich die These der affektiven Bindung an Architektur. Siehe dazu auch Knott et al. (2016) und Thrift (2004).
} 
kommt und diese zutiefst prägt, sind interreligiöse Dialoge Orte der Aushandlung der Grenzen zwischen religiösen Traditionen, der symbolischen Interessenverfolgung und des religiösen Lobbying (Nagel und Kalender 2014, S. 96-97; Nagel 2019). Multireligiöse Räume verleihen diesen Funktionen räumliche Sichtbarkeit, diskursive Prominenz und eine ästhetisch-materiale Form.

Wie eine Vielzahl von Studien zeigt, hat die Prominenz interreligiöser Dialoge innerhalb der letzten Jahrzehnte stark zugenommen, und zwar in dem Maße, in dem die Rolle von Religion für gesellschaftliche Integration diskursive Aufmerksamkeit erlangte und multiple Interessen beförderte (Körs et al. 2020). Dabei entstehen interreligiöse Dialoge sowohl aus lokalen Kontakten wie auch im top-down-Format aus staatlichen Initiativen heraus und kommen in lokalen Konfliktschlichtungs- und Mediationsverfahren, in öffentlichen auf Repräsentation abzielenden politischen Ritualen, in der Friedenspädagogik wie auch in der internationalen Diplomatie zur Anwendung (Griera und Fortzea 2013; Griera 2020). Während staatliche Interessen an interreligiösen Dialogen eng mit der Versicherheitlichung von Religion, insbesondere des Islam, im Kontext des Kampfs gegen Terrorismus zusammenhängen, nutzen religiöse Gruppen interreligiöse Plattformen zum Lobbying eigener Interessen (Religionsfreiheit, Beteiligung an politischen Entscheidungen) (Griera und Nagel 2018, S. 30; Martikainen 2013, S. 141).

In öffentlichen Diskursen werden multireligiöse Räume häufig als Orte der Begegnung, als Symbole interreligiöser Harmonie und Solidarität und als Beispiele für friedliche Koexistenz gepriesen, stehen sie doch für hierarchiefreie „Begegnungen auf Augenhöhe" und damit als Gegenmodelle zu einer von kollektiven Vorurteilen, gruppenbezogener Diskriminierung und Rassismen geprägten sozialen Umwelt (siehe Griera et al. 2019). Dabei wird häufig unterschlagen, dass die an multireligiösen Räumen beteiligten religiösen Akteur*innen - trotz der dem Management dieser Räume zugrundeliegenden deliberativen Verfahren - sehr unterschiedlich in den kulturellen Hierarchien westlicher Migrationsgesellschaften und deren urbanen Räumen positioniert sind. Diese Positionierungen haben einen maßgeblichen Einfluss auf die Art und Weise, wie multireligiöse Räume in der Gesellschaft wahrgenommen werden - sowohl bei den Bevölkerungsgruppen, die solche Räume (mehr oder weniger explizit) zu repräsentieren beanspruchen, wie auch jenen, die von ihnen ausgeschlossen werden.

Unser Beitrag adressiert die Frage, auf welche Weise sich diese Positionierungen in den Sichtweisen auf interreligiöse Beziehungen in urbanen Räumen dokumentieren, wie multireligiöse Räume wie das House of One von Berliner*innen unterschiedlicher religiöser Zugehörigkeit wahrgenommen werden, welche kulturellen Logiken in diesen Sichtweisen und Wahrnehmungen zum Ausdruck kommen und inwieweit sie das offizielle Skript des interreligiösen Dialogs (Tezcan 2012; AmirMoazimi 2011) in Frage stellen.

\section{Methodischer Zugang}

In unserer Studie kontrastieren und vergleichen wir die Perspektiven von drei unterschiedlich im Stadtraum positionierten Akteursgruppen: historisch dominante re- 
ligiöse Akteur*innen (evangelische Christ*innen), numerisch bedeutsame, aber gesellschaftlich stigmatisierte Gruppen (sunnitische Muslim*innen) und sowie Akteur*innen, die numerisch bedeutsam, jedoch nicht im House of One repräsentiert sind (Alevit*innen).

Das als Initiatorin am House of One beteiligte evangelische Christentum ist die in Berlin historisch dominante religiöse Gemeinschaft, ist im Stadtraum mit ihren solitären Kirchengebäuden besonders prominent und verfügt über ein etabliertes Netzwerk zivilgesellschaftlicher Organisationen, die routinemäßig mit staatlichen Stellen interagieren. Gleichwohl musste die evangelische Kirche Berlins in den letzten Jahrzehnten einen massiven Mitgliederschwund verzeichnen, was ihre gesellschaftliche Position tendenziell geschwächt hat. Die Rekonstruktion der protestantischen Perspektive basiert auf Interviews mit einem protestantischen Pfarrer, protestantischen Vertretern des House of One sowie zwei Beschäftigten eines protestantischen Stadtführungsunternehmens. ${ }^{10}$

Der sunnitische Islam ist in Berlin vor allem seit der in den 1960er-Jahren beginnenden Gastarbeitermigration aus der Türkei präsent und hat seitdem einen langwierigen Institutionalisierungsprozess durchlaufen. Gleichwohl sind Muslim*innen erheblichen gesellschaftlichen Restriktionen unterworfen, die sich an ihrer fortgesetzten marginalen stadtbildlichen Repräsentation (Jonker 1997), der Überwachung und teilweisen Stigmatisierung im Kontext von Anti-Terror-Kampagnen wie auch weiter verbreitetem behördlichen Misstrauen gegenüber muslimischen Akteur*innen (Schiffauer 2008) festmachen lassen. Er ist formal über die Organisation Forum für interkulturellen Dialog am House of One beteiligt, die selbst aber innerhalb des sunnitischen Feldes stark marginalisiert ist. Als empirisches Material wird hier eine Gruppendiskussion herangezogen, die mit Studierenden der islamischen Theologie durchgeführt wurde und einen entsprechend intellektuellen Duktus aufweist.

Die Präsenz des Alevitentums in Berlin entstand ebenfalls durch die Gastarbeitermigration aus der Türkei, wurde nachfolgend jedoch durch eine Reihe von antialevitischen Massakern in der Türkei und nachfolgenden Fluchtbewegungen wesentlich verstärkt. Diese Massaker und die Stigmatisierung des Alevitentums in der Türkei bestimmen auch die wechselseitigen Wahrnehmungen zwischen Alevit*innen und Sunnit*innen in Berlin. Während Alevit*innen im Stadtbild kaum wahrnehmbar sind, hat das Alevitentum in Deutschland in den letzten Jahren einen historisch einmaligen institutionellen Anerkennungsprozess durchlaufen (Sökefeld 2015). Alevit*innen werden durch staatliche Behörden tendenziell positiver wahrgenommen als Muslim*innen und haben auch ein vergleichsweise positives Bild vom deutschen Staat. Mit ca. 70.000 Mitgliedern ist das Alevitentum in Berlin eine der zahlenmäßig stärksten ethnisch-religiösen Gruppen, ist aber gleichwohl nicht im House of One vertreten. Interviewt wurde hier eine Gruppe alevitischer Gemeindemitglieder, die im Berliner Cemevi engagiert sind. ${ }^{11}$ Die interviewten muslimischen und alevitischen Gruppen sind selbst nicht aktiv am House of One beteiligt. Die

\footnotetext{
${ }_{10}$ Außer den Mitarbeiter*innen des Stadtführungsunternehmens waren damit alle protestantischen Interviewpartner Funktionäre.

${ }^{11}$ In beiden Gruppendiskussionen entschieden die kontaktierten Personen über die Zusammensetzung der Gruppen.
} 
Auswahl orientierte sich hier auf die Verankerung der religiösen Akteur*innen im Berliner Stadtraum Berlin. ${ }^{12}$

\section{Empirische Befunde: Blinde Flecken}

Der Beitrag basiert auf der Interpretation von individuellen problemzentrierten Interviews und Gruppendiskussionen mit Angehörigen dieser religiösen Gruppen. In der nachfolgenden Analyse identifizieren wir fünf „,blinde Flecken“ interreligiöser Dialoge und zeigen, auf welche Weise sich diese hier knapp skizzierten Positionen in den Perspektiven auf das House of One als Kristallisationspunkt interreligiöser Begegnungen dokumentieren.

\subsection{Blinder Fleck 1: Prominenz erzeugt Gegenreaktionen}

Ein Thema, das sich wie ein roter Faden durch die Diskussionen um das House of One zieht, ist der Aspekt der Sichtbarkeit bzw. Unsichtbarkeit von Religion im Stadtraum. Wie wir im Folgenden zeigen werden, verbindet sich für Protestant*innen Sichtbarkeit mit der ästhetischen und politischen Prominenz des House of One und mit der Hoffnung, dass diese zur Revitalisierung der gesellschaftlichen Relevanz des Protestantismus im Kontext sich beschleunigter Säkularisierungsprozesse beiträgt. Genau diese Prominenz des House of One wird hingegen von Muslim*innen und Alevit*innen, aus je unterschiedlicher Perspektive kritisiert, da sie die tatsächlich existierenden multiplen gesellschaftlichen Stigmatisierungen beider Gruppen im Alltagsleben zu überdecken droht. Statt um die Qualität tatsächlicher interreligiöser Beziehungen gehe es dann, so die Kritik, um am Leitbild des Multikulturalismus orientierte „Effekthascherei“, um inszenierte Diversität als entpolitisierte Außendarstellung und Performanz (siehe auch Walton 2015).

Bereits anhand einer metaphorischen Umschreibung des Projekts im Interview mit Pfarrer Köhler ${ }^{13}$ wird erkenntlich, dass Sichtbarkeit stellvertretend für Relevanz, Wirkung und Einfluss verstanden werden kann. Mit der Metapher des Leuchtturms wird hier auf einen Gebäudetyp verwiesen, dessen Funktion es ist, für Orientierung in der Dunkelheit zu sorgen, durch den leuchtenden Fixpunkt Menschen in die richtige Richtung zu lenken sowie einen markanten Ort hervorzuheben. Die spezielle Funktion des Leuchtturms tritt dann in den Vordergrund, wenn es um ihn herum dunkel ist. Überträgt man dieses Bild wieder auf den Kontext des House of One, als ein interreligiöses Bauprojekt in der Mitte der Hauptstadt Berlin, wird deutlich, welche Stellung und Aufgabe Religion und Kirche hier beigemessen wird: In einer weitestgehend als säkular wahrgenommenen Stadtkultur kann bzw. soll sie für Orientierung sorgen. Im übertragenen Sinne bringt somit die religiöse Dimension

\footnotetext{
12 Im Fortgang der Studie sollen auch die Perspektiven anderer Beteiligter, insbesondere von Jüd*innen, und stadträumlich bedeutender Unbeteiligter, etwa von Katholik*innen, in den Blick genommen werden. Ziel des Samplings der hier vorgelegten Analyse war nicht die Vollständigkeit, sondern das oben definierte Kontrastkriterium.

13 Alle Namen sind Pseudonyme, mit denen wir die Identität unserer Interviewpartner*innen wahren.
} 
Helligkeit in den als dunkel und verloren verstandenen urbanen Raum. Gleichzeitig wird in folgender Passage aus dem Interview mit Pfarrer Köhler deutlich, dass der Begriff Leuchtturm eine weitere Bedeutung beinhaltet:

Ich glaube auf der offiziellen Ebene macht das ganz schön Wirbel, also so die offiziell Verantwortlichen auf der Stadtverwaltungsebene und so, da sind natürlich alle Mitsponsoren und da macht das sehr viel Bewegung hab ich das Gefühl und ist auch über Berlin also in ganz Deutschland hinaus wirklich ein Leuchtturmprojekt sag ich mal, wo viele Leute auch hinkommen um zu gucken können wir das vielleicht in irgendeiner Weise auch für unsere Stadt übernehmen, dieses Projekt. Was habt ihr für Erfahrungen gemacht was würdet ihr uns raten. ${ }^{14}$

Köhler verwendete den Begriff Leuchtturmprojekt hier in einem Kontext, der ihn als Synonym für den Begriff Pilotprojekt deuten lässt, und wies darauf hin, dass es sich beim House of One um ein Projekt handelt, das „,viel Wirbel macht“, also neuartige und kontroverse Prozesse und Diskussionen anstößt. Zudem betonte er die Vorreiterrolle des Projekts sowohl auf behördlicher Ebene wie auch mit Blick auf die damit verbundene Vorbildfunktion für Projekte an anderen Orten. Implizit steckt hierin auch die Vorstellung, dass Berlin - bekannt für seinen innovativen und weltoffenen Ruf - den geeigneten Ort für ein solches Projekt darstellt und die Metropole der Provinz bezüglich Progressivität eine Spur voraus ist. Sichtbarkeit wird in diesem Kontext dementsprechend als aufsehenerregend, inspirierend und durchweg positiv bewertet. So meinte Köhler:

Was ich gesagt hab, so ein symbolischer Ausstrahlungsort in die Stadt hinein. Und wenn es da klappt und öffentlich sichtbar wird, dann klappt es auch woanders. Also ich glaube das ist die große Hoffnung daran dass man das so öffentlich auch zelebriert und sehr öffentlich macht auch das Ganze, damit man auch zeigen kann ,Leute das was ansonsten auch häufig in den Medien vorkommt, dass viele Konflikte nur daran liegen, weil wir Religionsvertreter uns nicht zusammenkriegen, das beweisen wir an diesem Ort: es geht auch anders““. ${ }^{15}$

Der Akzent liegt in dieser Darstellung auf der medialen und urbanen Sichtbarkeit des House of One, die mit der Mediendarstellung von religiösen Konflikten als Negativfolie kontrastiert wird und durch gelebte interreligiöse Kooperation auch positiv in den Stadtraum strahlen kann. Allerdings fokussiert - man könnte auch sagen: verengt - die Perspektive diese Sichtbarkeit auf die Rolle religiöser Eliten. Enthält das Zitat zwar die Hoffnung, dass Religionsvertreter*innen, anders als es der mediale Diskurs suggeriert, hier beispielhaft positiv zusammenwirken, rückt die Frage, mit welchen Vorstellungen religiöser Differenz sich Menschen tatsächlich im Alltag begegnen, dagegen in den Hintergrund. Dass in solchen Darstellungen damit also die Frage aktueller Problemlagen ,,vor Ort“ gar nicht berührt wird, ist immer wieder durch nichtchristliche Interviewpartner*innen kritisiert worden. Prominenz

14 Auszug aus dem Interview mit Pfarrer Köhler, geführt am 15.11.2019.

15 Auszug aus dem Interview mit Pfarrer Köhler, geführt am 15.11.2019. 
und Sichtbarkeit, bieten, wie wir im weiteren Verlauf zeigen werden, immer auch Angriffsfläche für Kritik.

\subsection{Blinder Fleck 2: Sichtbarkeit ist ein umkämpftes Gut und erzeugt Konkurrenz}

Diese durch mediale Prominenz und spektakuläre Architektur erzeugte Sichtbarkeit des House of One wurde in unseren Interviews kritisch mit der gefühlten Sichtbarkeit der eigenen religiösen Gemeinschaft im Alltag kontrastiert, steht sie doch auch für die Möglichkeit zur Wahrnehmung und Markierung religiöser Differenz in urbanen Räumen und die damit verbundene Anerkennung der Zugehörigkeit. Während die protestantischen Interviewten in diesem Zusammenhang anmerkten, dass das kulturelle Erbe der evangelischen Kirche in Vergessenheit zu geraten droht und entsprechende Symbole im Stadtraum immer häufiger übersehen werden, betonten Muslime, dass die Sichtbarkeit von Gotteshäusern je nach Stadt bzw. Stadtteil verschieden auffällig ist. Besonders Beispiele aus Süddeutschland und traditionell katholischen Regionen wurden herangezogen, um die Unsichtbarkeit und die in Berlin untergehenden Klänge (bspw. Kirchenglocken) zu kontrastieren.

Die Sichtbarkeit von Religion ist aber nicht lediglich auf die Wahrnehmung von Gebäuden und materialen Symbolen bezogen, sondern ebenso auf die Präsenz religiöser Praktiken im Stadtraum. Besonders betont wurde in diesem Zusammenhang die geteilte Beobachtung, dass selbst hohe Feiertage wie der Ramadan in Berlin im öffentlichen Raum kaum wahrnehmbar seien. Während hier bedauert wurde, dass die Praxis religiöser Feiern vorrangig hinter verschlossenen Türen stattfinde, ging es an anderer Stelle um die individuelle Praxis des Betens. Während das Beten im öffentlichen Raum einer Kleinstadt als befremdlich und irritierend wahrgenommen werden kann, so war sich die muslimische Gruppe einig, sorgt die Anonymität der Großstadt diesbezüglich für weniger potenziell störende Aufmerksamkeit. Dennoch führt auch in Städten wie Berlin der Mangel an Gebetsräumen in öffentlichen Institutionen, wie etwa Universitäten, aus Sicht der Muslime zu problematischen Formen der Sichtbarkeit. So meinte ein Interviewter: „Ich meine, so sieht man eben die Leute irgendwo in irgendwelchen Ecken beten, ich find das immer so ein bisschen unwürdig, also ich weiß nicht. [...] Das sind dann auch Protestgebete, sowas finde ich persönlich etwas heikel.“16 Ein anderes Gruppenmitglied fügte hinzu: „Es gefällt den Studenten selber ja auch nicht tatsächlich in den Ecken. Und andere fühlen sich dann gestört, weil man irgendeinen Bereich blockiert hat"“. ${ }^{17}$

Die Gruppenmitglieder fokussierten hier auf die praktischen Probleme, die sich aus religiösen Nutzungen urbaner Räume ergeben, und auch aus dieser Perspektive erscheint das House of One als problematisch, führt es doch von solchen Problemen systematisch weg. Während es symbolträchtige interreligiöse Begegnungen inszeniert, bleiben soziale Interaktionen, in denen praktische Raumprobleme zu bewältigen sind, weitgehend unartikuliert.

16 Auszug aus der Gruppendiskussion mit Muslim*innen, geführt am 20.01.2020.

17 Auszug aus der Gruppendiskussion mit Muslim*innen, geführt am 20.01.2020. 
Gleichzeitig machten die Interviewten die individualisierte Lebensführung sowie das Überangebot an Auswahlmöglichkeiten als Grund für Desinteresse und mangelndes Engagement im Feld urbaner Religiosität verantwortlich.

Nein aber wie sichtbar Religion ist hat sicher auch immer damit zu tun, wie sichtbar man es wahrnimmt oder ob man es wahrnehmen will. Und ich glaube man will Religion einfach auch gar nicht wahrnehmen, zumindest nicht anders wahrnehmen, als was weiß ich andere Lebenstrends oder so. ${ }^{18}$

An diesem kurzen Ausschnitt aus der Gruppendiskussion mit Muslim*innen wird deutlich, dass die Wahrnehmung von Religion im öffentlichen Raum nicht nur der Präsenz von religiösen Gebäuden, Praktiken und Symbolen bedarf, sondern auch die Bereitschaft der Stadtbevölkerung voraussetzt, diese zur Kenntnis zu nehmen. Sichtbarkeit ist dementsprechend nicht als einseitiges Phänomen zu betrachten, sondern vielmehr als gegenseitiger Prozess des sich Zeigens und des Sehens. Die hierin zum Ausdruck kommende Kritik am Desinteresse gegenüber der religiösen Dimension im Stadtraum Berlin zeigte sich auch in den Interviews mit den Vertreter*innen der Evangelischen Kirche. So wurde der Stadt Berlin von muslimischer wie auch evangelischer Seite attestiert, eine Stadt zu sein, die Religion von Grund auf mit Distanz und Skepsis gegenübersteht. Die Wahrnehmung Berlins als eine zwar multikulturelle, aber in der Öffentlichkeit überwiegend säkulare bzw. atheistische Stadt sorge auch dafür, so die Deutung an dieser Stelle, dass im alltäglichen Leben und Sich-Bewegen durch den urbanen Raum religiöse Symbole bewusst oder unbewusst übersehen werden.

Besonders deutlich wurde dies im Interview mit Vertreter*innen eines protestantischen Stadtführungsunternehmen, deren Angebot und Arbeit darin besteht, Berlin mit einem geschärften Blick auf religiöse Orte zu begehen. So verwiesen sie auf ihre Praxis, durch entsprechende Touren durch die Stadt eine fokussierte und aufmerksamere Wahrnehmung von historisch und religiös bedeutsamen Stätten und Symbolen zu entwickeln:

Und darüber hinaus gibt es ja so unglaublich viel in Berlin, und ich geh oder fahr jetzt mit dem Fahrrad auch wirklich völlig anders durch die Stadt und denk plötzlich ,,bist du schon so oft dran vorbeigefahren und hier ist die Gedenktafel für Hans Rosenthal“ oder auch Stolpersteine. Oder ,ach was ist denn das für eine Kirche“ oder ,,ach kuck mal hier ist irgendwie so eine ganz abgefahrene Religionsgemeinschaft" oder also so. ${ }^{19}$

Die Fülle an religiösen (Erinnerungs-)Orten steht, so wurde im weiteren Gespräch erläutert, nicht zuletzt damit in Zusammenhang, ,dass das Christentum eben die deutsche Geschichte sehr geprägt hat, also grade hier in Berlin. " ${ }^{20}$ Hier zeigt sich erneut, dass Sichtbarkeit und Wahrnehmung von Religionen im Stadtraum damit

\footnotetext{
18 Auszug aus der Gruppendiskussion mit Muslim*innen, geführt am 20.01.2020.

19 Auszug aus dem Interview mit einem protestantischen Stadtführungsunternehmen, geführt am 15.11.2019.

20 Auszug aus dem Interview mit einem protestantischen Stadtführungsunternehmen, geführt am 15.11.2019.
} 
verbunden sind, welche gesellschaftliche Position und Anerkennung der entsprechenden Religion beigemessen wird. So kann sich das protestantische Christentum auf seine lange Tradition und den damit einhergehenden materiellen Bestand sowie die kulturelle Einflussnahme berufen, während Vertreter*innen anderer Religionen, insbesondere des Islams, ihre Relevanz aus ihrer hohen Mitgliedschaft und ihrem Anspruch auf gesellschaftliche Teilhabe beziehen. Eine besondere Position hat hier das Judentum inne, das in Deutschland zwar eine lange Tradition und kulturellen Stellenwert hat, jedoch aufgrund der Vertreibung und Vernichtung im Nationalsozialismus heute in materialisierter Form vor allem durch Mahnmale und Erinnerungsorte sichtbar wird.

Dass Sichtbarkeit und Aufmerksamkeit neben Anerkennung und gesellschaftlicher Relevanz auch negative Effekte haben können, zeigt sich wiederum im Kontext von Marginalisierung und Diskriminierung von religiösen bzw. gesellschaftlichen Minderheiten. In der Gruppendiskussion mit Berliner Alevit*innen wurde betont, dass die Wahrnehmung von religiösen Symbolen und die damit verbundene Zuordnung von Glaubenszugehörigkeit ganz unmittelbare Konsequenzen für die Betroffenen bedeuten kann:

Abgesehen davon ist das für mich blödsinnig mit alevitischen oder mit christlichen oder mit jüdischen oder mit sonst irgendwelchen Symbolen spazieren zu gehen. Weil was sagst du damit aus? Du sagst damit aus ich gehöre zu einer Gruppe und anscheinend ist die Zugehörigkeit zu einer Gruppe von immenser Bedeutung, was zur Folge hat, dass du andere erstmal ausgrenzt. Und da hört schon der Spaß bei mir auf ne. Äußerliches Kennzeichen sagt aus, ich bin das, und das was ich bin ist gut, aber du gehörst nicht zu mir und das find ich schon sehr sehr schwierig. ${ }^{21}$

Die hier zitierte Stelle macht ganz deutlich, wie das Tragen religiöser Symbole und die damit verbundene Selbstmarkierung immer auch gleichzeitig ein Sichtbarmachen von Unterschieden und Hierarchien beinhaltet. Besonders im Falle der alevitischen Gemeinde birgt dieser Aspekt auch ein Moment von Diskriminierungserfahrung, die mit der Identifikation als Mitglied einhergeht. So verwiesen die Interviewten auf Stigmatisierungen von Seiten sunnitischer Muslime, wenn ihre Zugehörigkeit zum Alevitentum sichtbar wird. Die oben zitierte Kritik am Tragen religiöser Symbole ist dementsprechend aus ihrer eigenen Position innerhalb der Kultur- und Religionslandschaft Berlins herzuleiten. Darüber hinaus beinhaltet sie aber auch die Vorstellung, religiöse Selbstsymbolisierungen zielten auf Distinktion und Ausschluss. In diesem Zusammenhang wurde auch die Frage aufgeworfen, wer denn überhaupt durch ein Projekt wie das House of One an Sichtbarkeit und damit einhergehend an Macht und Bedeutung gewinne. Konträr zur Einstellung, dass das Tragen von religiösen Symbolen per se zu kritisieren ist, wurde an anderer Stelle im Interview problematisiert, dass im Falle von Anhänger*innen der Gülen-Bewegung das äußere Auftreten in die Irre leiten kann:

21 Auszug aus der Gruppendiskussion mit Alevit*innen, geführt am 18.07.2020. 
Die Gülens sind die größte Sekte, eine sehr durchdachte, eine hochintellektuelle Sekte. Alle Gülen-Anhänger sind auf dem Papier Akademiker. Das Interessante bei der Gülen-Bewegung ist, dass sie nach außen hin kaum wahrnehmbar sind, insbesondere deren Frauen nicht, weil sie fallen nicht auf. Die wenigsten von ihnen tragen Kopftücher, die Männer sind meistens richtig schicke Männer, sehr gepflegte Männer, fallen nicht durch einen Bart oder durch irgendwie, fallen überhaupt nicht auf. ${ }^{22}$

Im Zusammenhang mit einer ganz grundlegenden Kritik an der Gülen-Bewegung, insbesondere an ihren Strategien der Mitglieder-Akquise und Einflussnahme, wurde in der Gruppendiskussion betont, dass Selbstpräsentation und Haltung bzw. Lebensführung nicht übereinstimmen und dass Anhänger*innen der GülenBewegung „kaum wahrnehmbar“ seien. Während an anderer Stelle hervorgehoben wurde, dass die äußerliche Zuordnung zu einer Religion immer auch Exklusion und Hierarchisierung bedeutet, wurde hier hingegen auf die Gefahr einer optischen Täuschung hingewiesen. Durch das Fehlen typischer Erkennungsmerkmale von bekennend muslimischen Menschen, das Tragen des Kopftuchs bei Frauen und der Bart bei Männern, lassen sich Anhänger*innen der Gülen-Bewegung nur schwer als solche erkennen. Genau dieser Aspekt des Nicht-Auffallens stellte nach Ansicht der Interviewten die Gefahr dar, dass auch die religiöse Agenda der Bewegung nicht wahr- bzw. ernstgenommen wird und sie somit unbemerkt Einfluss ausüben können. Diese Perspektive birgt auch die Sorge bzw. Kritik, dass bei der Auswahl geeigneter Partnerverbände für das House of One nicht genau genug hingesehen und hinterfragt wurde, welche Ansichten und Haltungen sich hinter der auf den ersten Blick liberalen Gülen-Bewegung verbergen.

Sichtbarkeit, so wurde in den Interviews deutlich, kann je nach Kontext sowohl positiv als auch negativ gedeutet werden. Aus der Position einer traditionell etablierten Glaubensgemeinschaft, deren Einfluss sich jedoch zu verringern droht, verspricht ein „Leuchtturmprojekt" mit „Strahlkraft" den Erhalt von gesellschaftlicher Relevanz und kann als Element von ,place-keeping strategies“ (Becci et al. 2017) interpretiert werden. Aus der Perspektive einer Religion wie dem Islam, die sich zwar auf hohe Mitgliedszahlen berufen kann, gesellschaftlich aber nicht als gleichwertig angenommen wird, kann Sichtbarkeit als ein Faktor im Prozess der Anerkennung verstanden werden. Sie wurde in der Diskussion dann aber gerade nicht auf das House of One bezogen, sondern eben auf die Praktiken und Orte der eigenen Gemeinschaft. Neben den positiven Aspekten und Funktionen von Sichtbarkeit kann das Erkennen von Zugehörigkeit für weniger anerkannte Gruppen hingegen Exklusion bis hin zu Diskriminierung bedeuten.

Das Herstellen von Sichtbarkeit über ein Projekt wie das House of One muss dementsprechend nicht automatisch zu Gleichberechtigung führen, sondern kann gleichzeitig bereits marginalisierte Gemeinschaften und weniger prestigeträchtige Projekte in den Schatten stellen. Dies gilt in besonderer Weise für Muslim*innen,

22 Auszug aus der Gruppendiskussion mit Alevit*innen, geführt am 18.07.2020. 
die zwar formal über das Forum Dialog im House of One vertreten sind, das jedoch aufgrund seiner Assoziierung mit der Gülen-Bewegung von der Mehrheit der Berliner (türkischstämmigen) Muslim*innen abgelehnt wird. Damit verkehrt sich die Idee der Einbindung von Muslim*innen aus deren Sicht geradezu in ihr Gegenteil: Trotz ihrer formalen Einbindung sind sie nicht repräsentiert, sondern - aus ihrer Sicht - missrepräsentiert.

\subsection{Blinder Fleck 3: Interreligiöse Dialoge und interreligiöse Alltagsinteraktionen als divergierende Problemhorizonte}

Auf die Frage, wie die Interviewten interreligiöses Leben in Berlin wahrnähmen, wurde überwiegend auf Erfahrungen und Begegnungen im Alltag sowie auf soziale Projekte hingewiesen. So betonten die Interviewten, dass ebendiese Alltagsbegegnungen als beispielhaftes interreligiöses Miteinander zu deuten seien. Vor allem in den Interviews mit Protestant*innen fanden sich gehäuft Alltagsanekdoten, die als Positivbeispiele für eine gelebte Vielfalt fungierten. Besonders auffällig ist in diesem Zusammenhang, dass es die christlichen Interviewten positiv zu überraschen schien, von Muslim*innen in ihrem Glauben bzw. ihren religiösen Praktiken bestärkt zu werden. So erzählte der interviewte evangelische Pfarrer von der Begegnung mit einem muslimischen Taxifahrer:

Oder eine Taxifahrt in München wo mich ein Taxifahrer zu einer Hochzeit bringen musste, auch türkischer Herkunft, und der umarmt mich zum Schluss und sagt ,erzähl weiter von Gott, das ist in unserer Zeit ganz wichtig“. Und ich denk „wie geil ist das denn“. Ja, also von einem muslimischen Taxifahrer als Pfarrer umarmt zu werden sozusagen und der mich motiviert über Gott zu reden. ${ }^{23}$

Zunächst einmal handelt es sich hier um Dienstleistungsinteraktion: Der evangelische Pfarrer ist Fahrgast, der Mann türkischer Herkunft der Taxifahrer. Schon diese Konstellation macht hier typisch verteilte gesellschaftliche Positionen deutlich. Laut Hiebert et al. (2015) üben Serviceinteraktionen dieses Typs einen starken Einfluss auf interkulturelle Wahrnehmungen und Beziehungen aus. Durch das Erzählen dieser kleinen Alltagsgeschichte wird die eigene Offenheit zur Begegnung mit anders gläubigen Menschen betont, zugleich wurden auch Milieuunterschiede aufgemacht, die die hier erzählte, fast innige Begegnung umso außerordentlicher wirken lassen. Zentral für die stattfindende Verbrüderung ist die Religiosität als verbindende Gemeinsamkeit in einer vorwiegend säkularen Stadtgesellschaft. Statt einer Konkurrenz unterschiedlicher Glaubenszugehörigkeiten wurde hier die Einheit gegenüber einer ungläubigen Umwelt betont. Durch die in der Erzählung hervorgehobene positive Überraschung über den emotionalen Zuspruch des muslimischen Taxifahrers wird deutlich, dass der evangelische Pfarrer eine solch wohlwollende Reaktion nicht erwartet hatte. Zwar betont er indirekt seine eigene Unvoreingenommenheit, die Annäherung wurde hingegen von Seiten des Taxifahrers initiiert. Im hier erzählten Beispiel richtete sich die Bestärkung und Anerkennung dementsprechend von

23 Auszug aus dem Interview mit Pfarrer Köhler, geführt am 15.11.2019. 
der gesellschaftlich weniger anerkannten an die traditionell etablierte Position und durchkreuzte somit auch konventionelle Machtverhältnisse.

Die erzählte Passage dient als veranschaulichendes Beispiel dafür, wie direkt und unkompliziert interreligiöses Leben zwischenmenschlich und im Alltag stattfinden kann. Pfarrer Köhler betont im Interview wiederholt, dass er davon überzeugt sei, dass „dieses Interreligiöse auf der Basisebene ganz einfach“ sei. So etwa auch, als er berichtet, wie er einmal beim Einkauf einen türkischen Kioskbesitzer nebenbei zum Tag der offenen Tür in den Berliner Dom einlud, der dann tatsächlich kam:

Da kam dann einer der Domwärter und sagte ganz aufgeregt „Herr Köhler, da draußen stehen dreißig Leute und sagen, sie seien mit Ihnen verabredet.“ Ich geh raus, ist das der Kioskbesitzer mit seiner kompletten Familie und sagt ,wir wollten dich mal besuchen, du hast doch gesagt es ist Tag der offenen Tür, zeig uns mal den Berliner Dom." Alles Muslime! ${ }^{24}$

Alltägliche interreligiöse Begegnungen auf der „Basisebene“ werden aus der Position christlicher Vertreter*innen dementsprechend durchweg als positiv, weitestgehend unkompliziert und als wertvoller Beitrag zu einem friedlichen Miteinander der Religionen wahrgenommen.

Während in den Interviews mit den Protestant*innen größtenteils vernachlässigt wurde, dass interreligiöse Begegnungen im Alltag durchaus auch weniger friedlich verlaufen können bzw. Diskriminierungserfahrungen beinhalten können, betont Köhler Problemlagen interreligiöser Kontakte auf institutioneller Ebene. Als Beispiel führt er die kontroversen Diskussionen im Zuge der Rekrutierung der Projektpartner beim House of One an. Statt sich mit Dogmen aufzuhalten, so schlägt er vor, solle der Fokus vielmehr auf einem gelebten Miteinander und der Gemeinsamkeit eines Gottesglaubens liegen.

Auch in der Gruppendiskussion mit Muslim*innen wird eine Unterscheidung zwischen alltäglicher und institutionell initiierter Interreligiosität aufgemacht. Während soziale Projekte des gelebten Miteinanders positiv bewertet werden, stehen Projekte wie das House of One in der Kritik.

Eine Sache was ich mir auch denke, es ist wirklich merkwürdig dass es quasi ich hab auch das Gefühl dass es von oben kommt und eine elitäre Gruppe von paar Leuten dann sagt ,wir machen jetzt eine Moschee, und das braucht die Stadt et cetera“. Und dass es nicht so einen natürlichen Lauf nimmt, wie die Community merkt es fehlt etwas, und wir brauchen etwas und aus der Notwendigkeit heraus packen wir alle zusammen an, und machen etwas. ${ }^{25}$

Zentral ist in dieser Passage die Kontrastierung von einer gelebten Community, die als Expertin für ein praktisches Miteinander von gläubigen Menschen verstanden wurde, und der (inter-)religiösen Elite ohne ausreichendes Verständnis für die Wünsche und Bedürfnisse der Community-Mitglieder. Da Projekte, die ,,von oben“ beschlossen werden, nicht natürlich gewachsen sind, so die Perspektive der Interviewten, werden sie wenig Einfluss auf das gelebte Miteinander haben, sondern

24 Auszug aus dem Interview mit Pfarrer Köhler, geführt am 15.11.2019.

25 Auszug aus der Gruppendiskussion mit Muslim*innen, geführt am 20.01.2020. 
vielmehr auf der symbolischen Ebene verbleiben. Drastischer wurde an anderer Stelle formuliert, dass es sich bei einem Projekt wie dem House of One lediglich um Symbolpolitik handele, der eine „Instrumentalisierung von Religion“ zum Zwecke des Prestigegewinns zugrunde liege. „Von oben“ initiierte Projekte wie das House of One werden hier also vielmehr als ein künstliches Bündnis verstanden, das Nähe und Identität suggeriert, die stark von den ,realen“ Gegebenheiten abweichen bzw. von Seiten der Community gar nicht gewollt sind.

Als sinnvoller und bereits praktizierter Gegenentwurf verweisen Muslim*innen und Alevit*innen auf Initiativen, in denen sich Menschen verschiedener Glaubensrichtungen tatsächlich auf Augenhöhe und ganz unmittelbar begegnen und kennenlernen. So berichtete eine Teilnehmerin der Gruppendiskussion mit den Alevit*innen von folgender Initiative:

Also wir hatten damals ein Projekt gestartet, Brücken bauen für die Zukunft, Herr XY war der Leitgedanke, er hat das verschriftlicht, und ich war die Umsetzung und wir haben gesagt, wir verstehen uns als einen Teil einer pluralistischen Gesellschaft, wir haben Rechte, aber auch Pflichten. Das Cemevi wurde für alle umliegenden Schulen sozusagen als Lernort umgebaut, ja als Lernort umgebaut, Kinder aus bildungsfernen Elternhäusern, egal ob sie Christen, Atheisten, Hinduisten, oder weiß der Teufel was sie sind, hatten hier die Möglichkeit kostenlos Unterricht zu bekommen, die Mütter, hatten die Möglichkeit hier Deutschkurse zu machen, Väter und Mütter hatten die Möglichkeiten hier Unterricht zu bekommen. Was haben wir gesagt, Brücken bauen für die $\mathrm{Zu}$ kunft. Brücken kannst du nur bauen indem du alle inkludierst, jeder darf an deinem Tisch Platz haben. Hier hat nicht jeder Platz am Tisch, ist beschränkt. Das ist das eine, die bauen eine Brücke zueinander aber nicht zu uns, nicht zu den Anderen. ${ }^{26}$

Im Gegensatz zu einem elitären Projekt wie dem House of One, so die Interviewten, stehen bei alltäglichen interreligiösen Begegnungen das Interesse und die Bereitschaft zur Offenheit gegenüber Menschen unterschiedlichen Glaubens im Vordergrund. Das House of One gehe hingegen nicht weit genug, eine wirkliche Vielfalt zuzulassen, und Gruppen wie die alevitische Gemeinde werden nicht berücksichtigt. Zudem wurde darauf hingewiesen, dass bestehende interreligiöse Projekte häufig auf dem (ehrenamtlichen) Engagement der Gemeindemitglieder basieren und nur selten staatliche bzw. finanzielle Unterstützung oder Anerkennung bekämen. Vor diesem Hintergrund ist auch die folgende Passage aus einer Gruppendiskussion mit Mitgliedern eines muslimischen Dialog- und Bildungszentrums zu verstehen:

Es wird wahrscheinlich eine gute Ergänzung zu allem sein, aber ich würd's dabei belassen, letztendlich so wie du gesagt hast, also es ist eine Symbolik, es ist ein Zeichen, indem man quasi ein Zeichen setzt, aber die Arbeit, die wird ja schon seit Jahren so geführt quasi und die wird auch nach wie vor stattfinden, deswegen wird's da auch jetzt nicht unbedingt, oder die Arbeit wird jetzt nicht weniger werden dadurch. Ja, ich find's gut wie gesagt, ich hab mein per-

26 Auszug aus der Gruppendiskussion mit Alevit*innen, geführt am 18.07.2020. 
sönliches, also es ist wirklich jetzt nur mein persönlicher, also ich bin nicht unbedingt Fan davon, also wenn jetzt beispielshalber, ein Block weiter hier noch eine Moschee gebaut wird, nur als Beispiel, weil hier sind im Umkreis schon einige Moscheen, statt die weiter zu fördern, zu unterstützen, du hast es auch schon erwähnt, also man ist da auch ein bisschen auf sich alleine gestellt, man kriegt da keine Refinanzierung, staatlich gefördert, oder durch Projekte und so weiter. Dass man da einfach schaut, dass man die Menschen, die hier schon ohnehin Arbeit leisten, dass man da auch nochmal versucht irgendwie Unterstützung denen zu bieten. Ob jetzt finanziell, oder also durch jetzt Personal oder was auch immer. Ja, aber so wenn's jetzt nur rein um Symbolik geht, ist mir das, reicht mir persönlich nicht aus. ${ }^{27}$

Auch hier wird ein starker Kontrast zwischen der Symbolkraft des House of One und der interreligiösen (Alltags-)Praxis aufgemacht. Während der symbolische Charakter einer Moschee-Kirchen-Synagoge zwar als wichtig und gut bewertet wird, weist der Interviewte deutlich darauf hin, dass es nicht ausreiche, es bei einem Zeichen zu belassen. Man dürfe, so lässt sich dieser Abschnitt deuten, nicht erwarten, dass ein symbolisches Projekt unmittelbar für ein friedlich gelebtes Miteinander sorge. Vielmehr müsse die langjährige Arbeit vieler (teils ehrenamtlich tätiger) Menschen in selbstorganisierten, alltagsnahen Projekten und deren Beitrag zu einer friedlichen interreligiösen Stadtgemeinschaft anerkannt und gefördert werden. Die öffentliche Aufmerksamkeit, die das House of One erfährt, verleite, so die Sorge, nicht nur dazu, die Leistung weniger bekannter Akteur*innen zu übersehen, sondern berge auch die Gefahr einer ungleichmäßigen Verteilung von Fördergeldern und der darin zum Ausdruck kommenden Wertschätzung.

Die hier von Protestant*innen und Muslim*innen zugrunde gelegten Bewertungskriterien könnten kontrastreicher nicht sein. Während auf der einen Seite die symbolische (Strahl-)Kraft alles in den Hintergrund zu drängen und die Relevanz eines solchen Projektes ausreichend zu legitimieren scheint, geht es auf der anderen Seite vielmehr um die Bedürfnisse und Praktiken der (inter-)religiösen Community und die damit einhergehenden Zweifel daran, dass sich das House of One als Gebetsund Begegnungsstätte etablieren könne. Insgesamt stehen sich somit zwei grundlegende Deutungen entgegen: Einerseits wird das House of One als wichtiges Symbol für den interreligiösen Frieden angesehen, das sich durch seine Öffentlichkeit und Strahlkraft auch positiv auf das alltäglich gelebte Miteinander auswirken wird. Auf der anderen Seite wird hervorgehoben, dass das House of One in Konkurrenz mit bereits bestehenden Projekten auf der Basisebene tritt, sie aus der öffentlichen Wahrnehmung verdrängt und sich dadurch negativ auf deren Anerkennung und Förderung auswirkt.

27 Auszug aus der Gruppendiskussion mit Muslim*innen (2), geführt am 23.06.2020. 


\title{
5.4 Blinder Fleck 4: Interreligiöse Dialoge erzeugen Repräsentationsansprüche
}

Diese kritische Haltung gegenüber groß angelegten und von staatlicher Seite geförderten interreligiösen Projekten lässt sich in ganz anderer Weise in der Gruppendiskussion mit den Alevit*innen feststellen:

\begin{abstract}
Also ich finde, der Gedanke House of One steht im Kontrast zu der Vielfalt von Berlin. In Berlin gibt es laut, wenn ich mich jetzt nicht täusche, am Amerikahaus, an der Hardenbergstraße ist die Landeszentrale für politische Bildung und dort steht ein, dort ist so ein Tableau, auf dem steht, wenn ich mich, also auf die Zahl möcht ich mich nicht hundertprozentig festlegen, aber ich glaube 168 Glaubens- und Religionsgemeinschaften. House of One suggeriert mir eigentlich das, was die monotheistischen Weltreligionen wollen. Mit Ausnahme der Juden, weil da gehts nur über die Frau. Gibt es nur die drei auf der Welt? Und was sind die anderen, sind sie nichts wert? Wenn ich 168 minus drei, dann sind die 165 nichts? ${ }^{28}$
\end{abstract}

Während die Muslim*innen vor allem kritisierten, dass das Projekt House of One an der Lebensrealität vieler muslimischer Berliner*innen vorbeigeht, die Trias aus Judentum, Islam und Christentum hingegen nicht an sich in Frage gestellt wird, betonten die alevitischen Interviewten besonders die starke Divergenz zwischen der in Berlin gelebten Vielfalt an Religionen und der im House of One vertretenen Glaubensgemeinschaften. Dieser Fokus auf die drei ohnehin schon mächtigsten Weltreligionen beinhalte die Gefahr einer Verdrängung der religiösen Vielfalt in Bezug auf Wahrnehmung und Sichtbarkeit in der Öffentlichkeit. Insbesondere aus der Position des Alevitentums heraus, als zwar offiziell anerkannte, in der öffentlichen Aufmerksamkeit aber weitestgehend ignorierte Glaubensgemeinschaft, gewinnt dieser Aspekt zusätzlich an Bedeutung. Das Projekt House of One, so wurde hier beanstandet, widerspreche faktisch dem selbst auferlegten Anspruch, Symbol für die religiöse Vielfalt zu sein. Auch wenn die Repräsentant*innen des House of One immer wieder öffentlich betonen, es gehe ihnen nicht um die Repräsentation religiöser Traditionen bzw. der gesamten religiöse Vielfalt, werden sie doch permanent mit Repräsentationsansprüchen konfrontiert, die sie ob der Prominenz des Projekts kaum bewältigen können. Und auch die massive Förderung des Projekts mit öffentlichen Geldern macht es schwierig, sich solchen Repräsentationsansprüchen zu verweigern.

\subsection{Blinder Fleck 5: Interreligiöse Dialoge transportieren ambivalente Friedenssymboliken}

Protestant*innen sind häufig der Meinung, Projekte wie das House of One böten die Chance, den im Alltag ohnehin schon stattfindenden interreligiösen Dialog auf eine qualitativ neue Ebene zu heben, die für ein hohes $\mathrm{Ma} \beta$ an Öffentlichkeit und somit auch für den „Stadtfrieden“ sorge. So sagte ein evangelischer Pfarrer:

28 Auszug aus der Gruppendiskussion mit Alevit*innen, geführt am 18.07.2020. 
Da ist eine ganz große Hoffnung, die an diesem Ort auch steckt, dass Religionen für den Stadtfrieden wichtig sind. Und nicht nur für den Stadtunfrieden. So sehen wir uns ja als Christen ganz doll. Also wir sorgen für den Stadtfrieden. Das ist eine unserer größten Headlines, die wir hier vertreten. ${ }^{29}$

Demgegenüber steht die Kritik der muslimischen Interviewten, dass der Fokus auf symbolträchtige Projekte wie das House of One den gegenteiligen Effekt hervorrufe, nämlich bereits bestehende, weniger aufsehenerregende Projekte des interreligiösen Lebens in den Hintergrund zu drängen. Alevit*innen wiederum wandten gegen die Idee, dass ein Projekt wie das House of One symbolisch für den Frieden zwischen den Religionen stehe, ein, dass ein friedliches Miteinander ein solches Haus überflüssig machen würde: „Menschen, die friedlich sind, brauchen weder eine Religion noch ein Gebetshaus“". ${ }^{30}$ Das House of One steht aus dieser Perspektive dementsprechend, wenn überhaupt, für den Versuch, die existierenden Konflikte zwischen den Religionen zu besänftigen. Diese Haltungen, auf der einen Seite Stolz, auf der anderen Skepsis, so unser Argument, lassen sich aus den gesellschaftlichen Positionen der jeweiligen Glaubensgemeinschaften erklären. Während die Evangelische Kirche in Deutschland auf eine lange Tradition der politischen Einflussnahme zurückblicken kann, haben in Deutschland lebende Muslim*innen und andere Glaubensgemeinschaften weniger mächtige Dachverbände bzw. Vertretungen. Einig waren sich jedoch alle Interviewten, unabhängig von ihrer religiösen Zugehörigkeit, darin, dass die Vielfalt und das friedliche Zusammenleben von verschiedenen Glaubensgemeinschaften gefördert und unterstützt werden sollte. Problematisiert wurde dennoch, dass Vorhaben, die vorrangig symbolischen Charakters sind, reale Machtverhältnisse überdecken und sie eher reproduzieren als abzubauen.

\section{Zusammenfassung: Die Ambivalenz des Symbolischen}

In seiner Analyse architektonischer Designprozesse argumentierte der Soziologie Thomas Gieryn (2002, S. 42):

Design is both the planning of material things and the resolution of sometimes competing social interests. [...] The design process is simultaneously the representation of an artifact in graphic, verbal, or numerical form, and the enrollment or enlistment of those allies necessary to move the artifact toward material form.

Es steht außer Frage, dass sich die Wahrnehmungen und Bewertungen des House of One nach der tatsächlichen Errichtung des Gebäudes als materialem Artefakt und Kristallisationspunkt interreligiöser Beziehungen dynamisch weiterentwickeln werden. Und dennoch ist es bemerkenswert, auf welche Weise das Gebäude bereits vor seiner Errichtung gesellschaftliche Resonanz erzeugt, deren Artikulation unterschiedliche soziale Interessen erkennbar macht, die - so unser Argument - aufs Engste mit den unterschiedlichen gesellschaftlichen Positionen religiöser Gemein-

29 Auszug aus dem Interview mit Pfarrer Köhler, geführt am 15.11.2019.

30 Auszug aus der Gruppendiskussion mit Alevit*innen, geführt am 18.07.2020. 
schaften zusammenhängen. Drei Erkenntnisse des Beitrags sind in dieser Hinsicht zentral:

Erstens haben wir gezeigt, dass und auf welche Weise die Bezugnahmen auf das House of One und auf interreligiöse Dialoge kulturelle Hierarchien in Stadtgesellschaften sichtbar machen. Diese zeigen sich nicht zuletzt am Wert, der interreligiösen Dialogen und ikonischen Gebäuden wie dem House of One beigemessen wird. Protestant*innen tendierten dazu, interreligiöse Alltagsinteraktionen als unproblematisch wahrzunehmen, maßen aber dennoch ,hochkarätigen“ interreligiösen Projekten einen hohen Wert bei, der auch darin besteht, ihren eigenen Beitrag zur interreligiösen und gesellschaftlichen ,Friedenssicherung“ herauszustellen. Muslim*innen und Alevit*innen sehen, auf unterschiedliche Weise, interreligiöse Alltagsbegegnungen in einem kritischeren Licht, hinterfragen, ob ,hochkarätige“ - im Gegensatz zu lokal verankerten - interreligiöse Dialoge entsprechende Probleme adressieren, und bezweifeln deren Wert und Nutzen. Während das House of One die gleichberechtigte Zugehörigkeit im Stadtraum zum Ausdruck bringen und fördern soll - und dies in gewisser Weise auch tut - , macht es also paradoxerweise auch urbane Hierarchien sowie Ein- und Ausschlüsse stärker sichtbar.

Zweitens werden, wie unsere Analyse zeigt, dem House of One im Zuge kommunikativer Konstruktionsprozesse multiple und teilweise miteinander konkurrierende Bedeutungen zugewiesen. So wird das House of One einerseits als Ort der interreligiösen Begegnung und wechselseitigen Lernens, als Leuchtturm der Toleranz mit einer besonderen Strahlkraft und als steingewordenes Versprechen auf eine Zukunft friedlicher interreligiöser Koexistenz interpretiert. Andererseits wird es aber auch als exkludierend, elitär und von interreligiösen Alltagsbegegnungen abgehoben sowie sozial ,entbettet“" wahrgenommen und bewertet. Positive Bewertungen weisen dabei starke Bezüge zu offiziellen Diskursen auf, in denen das House of One semantisch als „multiculturalist place“ (Walton 2015, S. 107) fixiert sowie Dialog als per se wertvoll und gesellschaftlich folgenreich adressiert wird. Tendenziell kritische Bewertungen stellten, wie wir gezeigt haben, jedoch genau diese Annahmen in Frage. Damit ist klar, dass Architekturen eben keine Fixierungen vorab entworfenen kulturellen Sinns sind. Materialität, so könnte man schlussfolgern, schließt damit auch die Sinngebungsprozesse interreligiöser Dialoge nicht ab. Materialisierungen werden vielmehr zu Projektions- und Angriffsflächen von Kritik und diskursiven Auseinandersetzungen, die auch den Gegenstand selbst - Dialoge, die vermeintliche Monumentalität ihrer baulichen Form usw. - adressiert.

Drittens hat sich gezeigt, dass sich Divergenzen in der Wahrnehmung des House of One an unterschiedlichen, in der gesellschaftlichen Position der religiösen Akteur*innen gründenden Bewertungen von dessen Symbolkraft ansetzen. In der Perspektive der Akteur*innen steht der Begriff Symbol hier für (1) eine wegweisende Repräsentation eines Wertes (,Symbol für den Frieden“), (2) eine leere, substanzarme politische Geste (,Symbolpolitik“) und (3) eine in die Irre führende oder vereinseitigende Repräsentation. Es ist kein Zufall, dass gerade protestantische Vertreter*innen immer wieder auf die erste dieser Bedeutungen abheben, erlaubt ihnen doch das House of One das öffentliche Ausflaggen ihrer Initiierung und Beteiligung an einem politisch hochgradig relevanten Prozess, nämlich der Stärkung des gesellschaftlichen Zusammenhalts. Man sieht hier, wie sich Vertreter*innen der evangeli- 
schen Kirche in einer Art staatstragenden Verantwortung sehen, die auch die engen organisatorischen Verbindungen von Kirche und Staat widerspiegeln.

Umgekehrt stehen eben gerade Muslim*innen und Alevit*innen einer solchen Perspektive auf je eigene Weise kritisch gegenüber, da sich für sie automatisch die Frage stellt, inwieweit solche Projekte und Dialoge tatsächlich einen Einfluss auf die von ihnen im Alltagsleben gemachten Erfahrungen der Stigmatisierung und Marginalisierung haben. Die Ambivalenz der Symbolkraft von Architektur besteht dementsprechend darin, dass Projekte wie das House of One durchaus als kritische Entgegnungen auf die Marginalisierung religiöser Minderheiten gelesen werden, mit denen diese auch anerkannt werden, dass sie sich aber gleichzeitig dem Verdacht der „Schönfärberei“ und des Überdeckens der bestehenden Verhältnisse aussetzen. Und je mehr in interreligiösen Ritualen, Friedensgebeten und Events interreligiöse Toleranz heraufbeschworen wird, desto stärker erscheint - in den Augen marginalisierter Akteur*innen - der Kontrast zu einer Alltagswirklichkeit fortgesetzter wahrgenommener Stigmatisierung. Es ist diese Spannung zwischen der Symbolisierung des Versprechens auf eine noch zu schaffende und im Medium der Architektur gefasste tolerante Zukunft einerseits und der Symbolisierung von mit sozialen Ungleichheiten assoziierten religiösen Differenzen in der Gegenwart andererseits, die in den Wahrnehmungen des House of One zugespitzt zum Ausdruck kommt.

Die von uns zusammengetragenen Befunde decken sich teilweise mit anderen Studien, die auf in interreligiösen Dialogen zum Zuge kommende Exklusionstendenzen (Baumann und Tunger-Zanetti 2018), auf Grenzziehungs- und Grenzbearbeitungsmomente (Klinkhammer 2019) sowie auf die Effekte von interreligiösen Gebäuden auf nachbarschaftliche Beziehungen (Liljestrand 2018) aufmerksam gemacht haben. Gerade Liljestrands Studie hat auf die fundamentale Bedeutung der Einbettung multireligiöser Gebäude in nahräumliche Beziehungsgeflechte hingewiesen. Weitgehend unbeantwortet ist hingegen die Frage, inwieweit Architekturen mit ihren ästhetischen Codes und materialen Formen eigene Potenziale in den Umgang mit religiöser Vielfalt im urbanen Raum entfalten. Architekturen können affektive Bindungen stiften, wenn Gebäude von Menschen als positive Bezugspunkte wahrgenommen werden und wenn sie in der Lage sind, über die Mobilisierung emotionaler und kognitiver Ressourcen Atmosphären zu verstärken, innerhalb derer die Präsenz diverser religiöser Symbole, Praktiken und Identitäten als unproblematisch und thematisierbar erlebt wird. An Gebäuden wie dem House of One mit seiner zunächst sehr formalen architektonischen Sprache und seiner urbanen Prominenz wird man solche Dynamiken in besonderer Weise beobachten können.

Funding Open Access funding enabled and organized by Projekt DEAL.

Open Access Dieser Artikel wird unter der Creative Commons Namensnennung 4.0 International Lizenz veröffentlicht, welche die Nutzung, Vervielfältigung, Bearbeitung, Verbreitung und Wiedergabe in jeglichem Medium und Format erlaubt, sofern Sie den/die ursprünglichen Autor(en) und die Quelle ordnungsgemäß nennen, einen Link zur Creative Commons Lizenz beifügen und angeben, ob Änderungen vorgenommen wurden.

Die in diesem Artikel enthaltenen Bilder und sonstiges Drittmaterial unterliegen ebenfalls der genannten Creative Commons Lizenz, sofern sich aus der Abbildungslegende nichts anderes ergibt. Sofern das betreffende Material nicht unter der genannten Creative Commons Lizenz steht und die betreffende Handlung 
nicht nach gesetzlichen Vorschriften erlaubt ist, ist für die oben aufgeführten Weiterverwendungen des Materials die Einwilligung des jeweiligen Rechteinhabers einzuholen.

Weitere Details zur Lizenz entnehmen Sie bitte der Lizenzinformation auf http://creativecommons.org/ licenses/by/4.0/deed.de.

\section{Literatur}

Amir-Moazami, Schirin. 2011. Dialogue as a governmental technique: managing gendered Islam in Germany. Feminist review 98(1):9-27.

Astor, Avi, Victor Albert Blanco, und Rosa Martínez Cuadros. 2018. The politics of "tradition" and the production of Diasporic Shia religiosity. POMEPS Studies 32:32-38.

Bartmanski, Dominic, und Jeffrey Alexander. 2012. Materiality and meaning in social life: toward an iconic turn in cultural sociology. In Iconic power: materiality and meaning in social life, Hrsg. Jeffrey Alexander, Dominik Bartmanski, und Bernhard Giesen, 1-15. Basingstoke: Palgrave Macmillan.

Baumann, Martin, und Andreas Tunger-Zanetti. 2018. Constructing and representing the new religious diversity with old classifications: "World Religions" as an excluding category in interreligious dialogue in Switzerland. In The critical analysis of religious diversity, Hrsg. L. Kühle, J. Borup, und W. Hoverd, 179-207. Leiden: Brill.

Becci, Irene, Marian Burchardt, und Maria Giorda. 2017. Religious super-diversity and spatial strategies in two European cities. Current Sociology 65(1):73-91.

Berger, Peter L. 2014. The many altars of modernity: toward a paradigm for religion in a pluralist age. Boston/Berlin: De Gruyter.

Brighenti, Andrea. 2007. Visibility: a category for the social sciences. Current Sociology 55(3):323-342.

Brubaker, Rogers. 2017. Between nationalism and civilizationism: the European populist moment in comparative perspective. Ethnic and Racial Studies 40(8):1191-1226.

Burchardt, Marian. 2017. Infrastrukturen des Religiösen: Materialität und urbane Ordnungsregime. In Architekturen und Artefakte, Hrsg. U. Karstein, T. Schmidt-Lux, 233-250. Wiesbaden: Springer VS.

Burchardt, Marian, und Irene Becci. 2016. Religion and superdiversity: an introduction. New Diversities 18(1):1-7.

Burchardt, Marian, und Mar Griera. 2019. To see or not to see: explaining intolerance against the "Burqa" in European public space. Ethnic and Racial Studies 42(5):726-744.

Cesari, Jocelyne. 2005. Mosque conflicts in European cities: introduction. Journal of Ethnic and Migration Studies 31(6): 1015-1024.

Coole, Diana, und Samantha Frost. 2010. Introducing the new materialisms. In New Materialisms: ontology, agency, and politics, Hrsg. Diana Coole, Samantha Frost, 1-43. Durham, London: Duke University Press.

DeNora, Tia. 2000. Music in everyday life. Cambridge: Cambridge University Press.

Duttweiler, Stefanie. 2017. Entschärfte Säkularisierung - gezähmte Religiosität - (multi-) religiöse Räume als räumliche Materialisierungen der Postsäkularisierung. Geographica Helvetica 72(3):283-294.

Fischer, Joachim. 2017. Gebaute Welt als schweres Kommunikationsmedium der Gesellschaft. Architektur und Religion aus architektursoziologischer Perspektive. In Architekturen und Artefakte, Hrsg. U. Karstein, T. Schmidt-Lux, 49-69. Wiesbaden: Springer VS.

Garbin, David. 2013. The Visibility and Invisibility of Migrant Faith in the City: Diaspora Religion and the Politics of Emplacement of Afro-Christian Churches. Journal of Ethnic and Migration Studies 39(5):677-696.

Gieryn, Thomas F. 2002. What buildings do. Theory and Society 31(1):35-74.

Goffman, Erving. 1959. Stigma: notes on the management of spoiled identity. New York: Simon and Schuster.

Göle, Nilüfer. 2011. The public visibility of islam and European politics of resentment: the minaretsmosques debate. Philosophy \& Social Criticism 37(4):383-392.

Griera, Mar. 2019. Interreligious events in the public space: performing togetherness in times of religious pluralism. In Interreligious relations and the negotiation of ritual boundaries, Hrsg. M. Moyaert, 35-55. Cham: Palgrave Macmillan.

Griera, Mar. 2020. Governing Religious Diversity Through Interreligious Initiatives: Affinities, Ambiguities and Tensions. In A. Körs, W. Weisse, und W. Jean-Paul, Hrsg. Religious Diversity, Interreligious Dialogue, 89-102. Cham: Springer. 
Griera, Mar, und Marian Burchardt. 2020. Urban regimes and the interaction order of religious minority rituals. Ethnic and Racial Studies 44(10):1712-1733.

Griera, Mar, und Maria Forteza. 2013. New actors in the governance of religious diversity in European cities. In Religious actors in the public sphere: means, objectives, and effects, Hrsg. J. Haynes, A. Hennig, 113-131. New York: Routledge.

Griera, Mar, und Alexander-Kenneth Nagel. 2018. Interreligious relations and governance of religion in europe: introduction. Social Compass 65(3):301-311.

Griera, Mar, Marian Burchardt, und Astor Avi. 2019. European identities, heritage, and the iconic power of multi-religious buildings: cordoba's mosque cathedral and Berlin's house of one. In Volume 10: interreligious dialogue, Hrsg. G. Giordan, A.P. Lynch, 13-31. Leiden: Brill.

Hafner, Johann, Helga Völkening, und Irene Becci. 2018. Glaube in Potsdam. Bd. I. Baden-Baden: Ergon.

Hiebert, Daniel, Jan Rath, und Steven Vertovec. 2015. Urban markets and diversity: towards a research agenda. Ethnic and Racial Studies 38(1):5-21.

Jonker, Gerdien. 1997. Die islamischen Gemeinden in Berlin zwischen Integration und Segregation. In Zuwanderung und Stadtentwicklung, Hrsg. H. Häußermann, I. Oswald, 347-364. Wiesbaden: VS.

Karstein, Uta. 2013. Konflikt um die symbolische Ordnung. Genese, Struktur und Eigensinn des religiösweltanschaulichen Feldes in der DDR. Würzburg: Ergon.

Karstein, Uta, und Thomas Schmidt-Lux. 2017. Architekturen und Artefakte: Zur Materialität des Religiösen. Wiesbaden: Springer VS.

Keane, Webb. 2007. Christian moderns: freedom and fetish in the mission encounter. Berkeley: University of California Press.

Klinkhammer, Gritt. 2019. Der interreligiöse Dialog als Boundary Work. Zeitschrift für Religionswissenschaft 27(1):78-102.

Knoblauch, Hubert. 2021. Urbi et Orbi: pope Benedict's visit to Berlin and the emplacement of communicative events. In Urban religious events: public spiritualities in contested spaces, Hrsg. P. Bramadat, M. Griera, J. Martinez-Arino, und M. Burchardt, 143-158. London: Bloomsbury.

Knott, Kim. 2015. Walls and other unremarkable boundaries in south London: impenetrable infrastructure or portals of time, space and cultural difference? New Diversities 17(2):15-34.

Knott, Kim, Volkhard Krech, und Birgit Meyer. 2016. Iconic religion in urban space. The Journal of $O b$ jects, Art and Belief 12(2):123-136.

Knowles, Caroline. 2013. Nigerian London: re-mapping space and ethnicity in superdiverse cities. Ethnic and Racial Studies 36(4):651-669.

Körs, Anna, Wolfram Weisse, und Jean-Paul Willaime. 2020. Religious diversity and interreligious dialogue. Berlin: Springer.

Kuppinger, Petra. 2014. Mosques and minarets: conflict, participation and visibility in German cities. Anthropological Quarterly 87(3):793-818.

Lamont, Michèle, und Virág Molnár. 2002. The study of boundaries in the social sciences. Annual Review of Sociology 28(1):167-195.

Lanz, Stephan. 2016. The born-again Favela: the urban informality of pentecostalism in Rio de Janeiro. International Journal of Urban and Regional Research 40(3):541-558.

Latour, Bruno. 2001. Eine Soziologie ohne Objekt? Berliner Journal für Soziologie 11(2):237-252.

Lefebvre, Henri. 2016. Das Recht auf Stadt: Nautilus Flugschrift. Hamburg: Edition Nautilus.

Liljestrand, Johan. 2018. How interreligious buildings influence interreligious neighbourhood relations. In Religion and dialogue in the city: case studies on interreligious encounter in urban community and education, Hrsg. J. Ipgrave, T. Knauth, A. Körs, D. Vieregge, und M. von der Lippe, 159-180. Münster / New York: Waxmann.

Löw, Martina. 2001. Raumsoziologie. Berlin: Suhrkamp.

Martikainen, Tuomas. 2013. Multilevel and Pluricentric Network Governance of Religion. In Religion in the Neoliberal Age: Political Economy and Modes of Governance, Hrsg. F. Gauthier, T. Martikainen, 141-154. Farnham: Ashgate.

Meyer, Birgit. 2011. Mediation and immediacy: sensational forms, semiotic ideologies and the question of the medium. Social Anthropology 19(1):23-39.

Nagel, Alexander-Kenneth. 2019. Enacting diversity: boundary work and Performative dynamics in interreligious activities. In Volume 10: interreligious dialogue, Hrsg. G. Giordan, A.P. Lynch, 111-127. Leiden: Brill.

Nagel, Alexander-Kenneth, und Mehmet Kalender. 2014. The many faces of dialogue: driving forces for participating in interreligious activities. In Religions and dialogue, Hrsg. K. Amirpur, W. Weisse, 85-98. Münster: Waxmann. 
Schiffauer, Werner. 2008. Parallelgesellschaften: Wie viel Wertekonsens braucht unsere Gesellschaft? Für eine kluge Politik der Differenz. Bielefeld: transcript Verlag.

Schmidt-Lux, Thomas. 2017. Kirche und Aula zugleich? Eine Gebäudeinterpretation des Leipziger Paulinums. In Architekturen und Artefaket: Zur Materialität des Religiösen, Hrsg. U. Karstein, T. SchmidtLux, 121-144. Wiesbaden: Springer VS.

Sklair, Leslie. 2010. Iconic architecture and the culture-ideology of consumerism. Theory, Culture \& Society 27(5):135-159.

Sökefeld, Martin. 2015. Aleviten in Deutschland: Identitätsprozesse einer Religionsgemeinschaft in der Diaspora. Bielefeld: transcript.

Steets, Silke. 2015. Der sinnhafte Aufbau der gebauten Welt: Eine Architektursoziologie. Berlin: Suhrkamp.

Steets, Silke. 2017. Seductive atmospheres, conflicting symbols: religious landmark buildings in diverse societies. Eurostudia 12(1):125-135.

Tezcan, Levent. 2012. Das muslimische Subjekt: Verfangen im Dialog der Deutschen Islam Konferenz. Konstanz: Konstanz University Press.

Thrift, Nigel. 2004. Intensities of feeling: towards a spatial politics of affect. Geografiska Annaler: Series B, Human Geography 86(1):57-78.

Vertovec, Steven. 2007. Super-diversity and its Implications. Ethnic and racial studies 30(6):1024-1054.

Walton, Jeremy F. 2015. Architectures of interreligious tolerance: the Infrastructural politics of place and space in Croatia and Turkey. New Diversities 17(2):103-117.

Zubrzycki, Geneviève. 2016. Beheading the saint: nationalism, religion, and secularism in Quebec. Chicago: University of Chicago Press. 